\title{
Perturbation theory for dual semigroups II. Time-dependent perturbations in the sun-reflexive case
}

\author{
Ph. Clément \\ Delft University of Technology, Department of Mathematics and Informatics, \\ Julianalaan 132, Postbus 356, 2600 AJ Delft, The Netherlands

\section{O. Diekmann} \\ Centre for Mathematics and Computer Science, P.O. Box 4079, \\ 1009 AB Amsterdam, The Netherlands \\ and \\ Institute of Theoretical Biology, University of Leiden, Groenhovenstraat 5, \\ 2311 BT Leiden, The Netherlands

\section{Gyllenberg} \\ Helsinki University of Technology, Department of Mathematics and Systems \\ Analysis, SF-02150 Espoo, Finland
}

\section{H. J. A. M. Heijmans}

Centre for Mathematics and Computer Science, P.O. Box 4079, 1009 AB Amsterdam, The Netherlands

$$
\text { and }
$$

\section{H. R. Thieme}

Sonderforschungsbereich 123, Universität Heidelberg, Im Neuenheimer Feld 294, D-6900 Heidelberg, Bundesrepublik Deutschland

(MS received 4 June 1987. Revised MS received 27 October 1987)

\section{Synopsis}

We consider time-dependent perturbations of generators of strongly continuous semigroups on a Banach space. The perturbations map the Banach space into a bigger space, which is the second dual of the original space in a specific semigroup sense. Using the theory of dual semigroups we show that the solutions of a generalised variation-of-constants formula define an evolutionary system. We investigate continuity and differentiability properties of this evolutionary system and its dual system and examine in what sense the perturbed generator and its adjoint generate these evolutionary systems. It is shown that the results apply naturally to retarded functional differential equations and age structured population dynamics.

\section{Introduction}

In a recent paper [5] we considered the abstract Cauchy problem

$$
\left.\begin{array}{l}
\frac{d u(t)}{d t}=A_{0} u(t)+B u(t), \quad t>0, \\
u(0)=x \in X,
\end{array}\right\}
$$


where $A_{0}$ is the infinitesimal generator of a $C_{0}$-semigroup $\left\{T_{0}(t)\right\}_{t \geqq 0}$ of bounded linear operators on a Banach space $X$, and $B$ is a bounded linear operator from $X$ not into $X$ but into a bigger space $X^{\odot *}$. Here $X^{\odot}=\overline{D\left(A_{0}^{*}\right)}$ is the largest subspace of $X^{*}$ (the normed dual of $X$ ) on which the dual semigroup $\left\{T_{0}^{*}(t)\right\}_{t \geqq 0}$ is strongly continuous and $X^{\odot *}$ is the dual of $X^{\odot}$. The semigroup $T_{0}^{*}(t)$ leaves $X^{\odot}$ invariant so by restricting $\left\{T_{0}^{*}(t)\right\}_{t \geqq 0}$ to $X^{\odot}$ one can define the strongly continuous semigroup $\left\{T_{0}^{\odot}(t)\right\}_{t \geqq 0}$ on $X^{\odot}$ with generator $A_{0}^{\odot}$ (the part of $A_{0}^{*}$ in $X^{\odot}$ ). If the canonical injection $j: X \rightarrow X^{\odot *}$, defined by $\left\langle x^{\odot}, j(x)\right\rangle=\left\langle x, x^{\odot}\right\rangle$, maps $X$ onto $X^{\odot \odot}, X$ is called sun-reflexive (with respect to $A_{0}$ ) and $X^{\odot \odot}$ is identified with $X$. For more details on dual semigroups, see $[4,15]$. Those results needed in the present paper are summarised in [5].

In [5] we showed that if $X$ is sun-reflexive, then the variation-of-constants formula

$$
T(t) x=T_{0}(t) x+\int_{0}^{t} T_{0}^{\odot *}(t-\tau) B T(\tau) x d \tau, \quad x \in X
$$

uniquely defines a $C_{0}$-semigroup $\{T(t)\}_{t \geqq 0}$ on $X$ with generator $A=A_{0}^{\odot *}+B$, $D(A)=\left\{x \in D\left(A_{0}^{\odot *}\right): A_{0}^{\odot *} x+B x \in X\right\}$. Thus $T(t) x$ can be viewed as a mild solution to (1.1), where one actually should replace $A_{0}$ by $A_{0}^{\odot *}$.

In this paper we shall generalise the results in [5] to the case of nonautonomous perturbations where $B$ is not fixed but depends on $t$. We show that if $\{B(t)\}_{t \in[0, T]}$ is a strongly continuous family of bounded linear operators from $X$ into $X^{\odot *}$, then the variation-of-constants formula

$$
U(t, s) x=T_{0}(t-s) x+\int_{s}^{t} T_{0}^{\odot *}(t-\tau) B(\tau) U(\tau, s) x d \tau, \quad x \in X, \quad s<t,
$$

determines an evolutionary system on $X$. We investigate whether it is possible, as in the autonomous case, to extend $\{U(t, s)\}_{0 \leqq s \leqq r \leqq T}$ to $X^{\odot *}$ by taking adjoints and restrictions. The answer to this question depends on continuity properties of $\{U(t, s)\}_{0 \leqq s \leqq t \leqq T}$, which in turn depend on continuity properties of the mapping $t \rightarrow B(t)$.

Guided by the autonomous case one expects that $\{U(t, s)\}_{0 \leqq s \leqq t \leqq T}$ is in some sense generated by the family $\left\{A_{0}^{\odot *}+B(t)\right\}_{t \in[0, T]}$. We shall therefore investigate under what conditions and in what sense $U(t, s)$ is a solution of the forward problem

$$
\left.\begin{array}{l}
\frac{\partial}{\partial t} U(t, s)=\left(A_{0}^{\odot *}+B(t)\right) U(t, s), \quad s<t \leqq T, \\
U(s, s)=I,
\end{array}\right\}
$$

and of the backward problem

$$
\left.\begin{array}{l}
\frac{\partial}{\partial s} U(t, s)=-U^{\odot *}(t, s)\left(A_{0}^{\odot *}+B(s)\right), \quad 0 \leqq s<t, \\
U(t, t)=I .
\end{array}\right\}
$$

So the theory of this paper includes existence, uniqueness, representation and regularity of solutions as well as computation and representation of adjoints. 
One of the main applications we have in mind concerns retarded functional differential equations

$$
\left.\begin{array}{l}
\dot{x}(t)=\int_{0}^{1} x(t-\theta) d_{\theta} \zeta(t, \theta), \quad t>s, \\
x(s+\theta)=\phi(\theta), \quad \theta \in[-1,0],
\end{array}\right\}
$$

where for each $t, \zeta(t, \cdot)$ is a normalised function of bounded variation on $[0,1]$, and $\phi$ is a given initial function, defined and continuous on $[-1,0]$. The subscript $\theta$ of $d$ in (1.6) means that $\theta$ is the integration variable. In his book [14, Chap. 6], Hale treats equation (1.6) using a variation-of-constants formula involving the so-called fundamental matrix, which by definition is the solution of (1.6) with discontinuous initial condition $x(t)=0, s-1 \leqq t<s, x(s)=I$ ( $=$ the identity matrix). Since Hale treats problem (1.6) in the space $C$ of continuous functions, the fundamental matrix does not fit naturally into the framework of evolutionary systems on $C$. To obtain a variation-of-constants formula of the type (1.3) for the problem (1.6) we view (1.6) as a non-autonomous perturbation problem, where the equation $\dot{x}(t)=0$, regarded as a delay equation, plays the role of the unperturbed equation. The autonomous version of (1.6) has already been discussed in this framework by Diekmann [10]. It turns out that $X^{\odot *}=R^{n} \times$ $L^{\infty}\left([-1,0] ; R^{n}\right)$ and $B(t) \phi=(\langle\phi, \zeta(t, \cdot)\rangle, 0)$, see Examples 3.3 and 4.10 for more details. Of course the fundamental matrix solution is well-defined in $R^{n} \times$ $L^{\infty}\left([-1,0] ; R^{n}\right)$. In this manner we find a framework which allows us to rewrite parts of [14] in a functional analytic spirit.

Another important application concerns the dynamics of age-structured populations. The autonomous case was treated in detail in [5]. The results of the present paper apply to situations where fecundity depends explicitly on time.

In both the aforementioned applications the operator $B(t)$ or $B^{*}(t)$ has a fixed finite dimensional range. In Section 6 we will systematically investigate the special properties which derive from such a range condition. Thus it appears that the present functional analytic framework unifies at least the theory of delay equations and of age dependent population problems. Applications to parabolic equations are under study and the results obtained so far are promising.

Abstract evolutionary systems and their connection with Cauchy problems have been studied by several authors, beginning with Kato [16] and including Tanabe [21], Belleni-Morante [3], Dorroh and Graff [12], Pazy [19] and Kellermann [17]. The theory of semigroups on sun-reflexive Banach spaces has been applied by Amann [1].

We close this section with some remarks on notation and terminology. If $Z$ is a Banach space and $Z^{*}$ its dual, we use $\left\langle z, z^{*}\right\rangle$ and $\left\langle z^{*}, z\right\rangle$ interchangeably to denote the value of $z^{*}$ at $z$, whenever $z \in Z, z^{*} \in Z^{*}$. Elements of $X$ and $X^{\odot *}$ will mostly be written to the left and elements of $X^{*}$ and $X^{\odot}$ to the right in the duality brackets. Although the weak*-topology will play an important role, all topological concepts relating to a Banach space or its dual will refer to their respective norm topologies, unless the contrary is explicitly stated.

Integrals of functions with values in a dual Banach space are regarded as weak* Riemann integrals. This is sufficient for our purposes since we shall only 
encounter integrals of weak*-continuous functions. So if $Z$ is a Banach space and $t \rightarrow z^{*}(t)$ is continuous from $[a, b]$ to $Z^{*}$ equipped with its weak ${ }^{*}$-topology, then $\int_{a}^{b} z^{*}(\tau) d \tau$ is defined as the unique element of $Z^{*}$ satisfying $\left\langle z, \int_{a}^{b} z^{*}(\tau) d \tau\right\rangle=$ $\int_{a}^{b}\left\langle z, z^{*}(\tau)\right\rangle d \tau$ for all $z \in Z$.

If $Y$ and $Z$ are Banach spaces, then $\mathscr{B}(Y, Z)$ denotes the Banach space of all bounded linear operators from $Y$ to $Z$ and $\mathscr{B}(Y, Y)$ is abbreviated to $\mathscr{B}(Y)$.

\section{Evolutionary systems and the variation-of-constants formula}

Let $X$ be a Banach space and let $\left\{T_{0}(t)\right\}_{t \geqq 0}$ be a $C_{0}$-semigroup with generator $A_{0}$ on $X$ and assume that $X$ is sun-reflexive with respect to $A_{0}$. Let $T>0$ and let $\{B(t)\}_{t \in[0, T]}$ be a family of bounded linear operators from $X$ to $X^{\odot *}$. We assume that $\{B(t)\}_{t \in[0, T]}$ is strongly continuous, that is, for each $x \in X$, the mapping $t \rightarrow B(t) x$ is continuous from $[0, T]$ to $X^{\odot *}$. These basic assumptions will be valid throughout the paper.

Definition 2.1. Let $T>0$ and let $\Delta:=\left\{(t, s) \in R^{2}: 0 \leqq s \leqq t \leqq T\right\}, \quad \Delta^{*}:=$ $\left\{(s, t) \in R^{2}: 0 \leqq s \leqq t \leqq T\right\}$. A two-parameter family $\{U(t, s)\}_{(t, s) \in \Delta}$ of bounded linear operators on a Banach space $X$ is called a forward evolutionary system on $X$ if the following two conditions are satisfied:

(i) $U(s, s)=I$ (the identity), $0 \leqq s \leqq T$,

(ii) $U(t, r) U(r, s)=U(t, s), 0 \leqq s \leqq r \leqq t \leqq T$.

A two-parameter family $\{V(s, t)\}_{(s, t) \in \Delta^{*}}$ of bounded linear operators on $X$ is called a backward evolutionary system if

(i) $*(t, t)=I, 0 \leqq t \leqq T$;

(ii) $^{*} V(s, r) V(r, t)=V(s, t), 0 \leqq s \leqq r \leqq t \leqq T$.

When no confusion can arise, or when a statement holds for both types of systems, we shall omit the adjective forward or backward. An evolutionary system $\{U(t, s)\}_{(t, s) \in \Delta}\left[\{V(s, t)\}_{(s, t) \in \Delta^{*}}\right]$ is said to be strongly continuous if for every $x \in X$ the mapping $(t, s) \rightarrow U(t, s) x[(s, t) \rightarrow V(s, t) x]$ is (jointly) continuous from $\Delta\left[\Delta^{*}\right]$ to $X$.

Let $\{U(t, s)\}_{(t, s) \in \Delta}$ be a forward evolutionary system and, for every $(t, s) \in \Delta$, let $U(t, s)^{*} \in \mathscr{B}\left(X^{*}\right)$ be the adjoint operator of $U(t, s)$. Define (nota bene the position of the star) $U^{*}(s, t)=U(t, s)^{*}$ for $0 \leqq s \leqq t \leqq T$. Since

$$
\begin{aligned}
\left\langle x, U^{*}(s, r) U^{*}(r, t) x^{*}\right\rangle & =\left\langle U(t, r) U(r, s) x, x^{*}\right\rangle \\
& =\left\langle U(t, s) x, x^{*}\right\rangle=\left\langle x, U^{*}(s, t) x^{*}\right\rangle,
\end{aligned}
$$

we see that $\left\{U^{*}(s, t)\right\}_{(s, t) \in \Delta^{*}}$ is a backward evolutionary system. We call it the

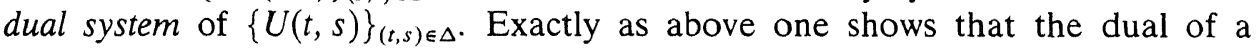
backward system is a forward system.

Our first objective is to show that the variation-of-constants formula (1.3) defines an evolutionary system on $X$ and to derive some basic properties of the dual system. Suppose $\tau \rightarrow U(\tau, s) x$ is continuous from $[s, T]$ to $X$. Then the integral term on the right-hand side of (1.3) is a well-defined element of $X^{\odot *}$. But for (1.3) to hold, the integral should belong to $X$. The following lemma implies that this is indeed the case. 
Lemma 2.2. Let $f: \Delta \rightarrow X^{\odot *}$ be continuous. Then the function $F$ defined by

$$
F(t, s)=\int_{s}^{t} T_{0}^{\odot *}(t-\tau) f(\tau, s) d \tau, \quad(t, s) \in \Delta
$$

is continuous from $\Delta$ to $X$.

The proof is very similar to that of [5, Theorem 3.2] and is therefore omitted. The proof actually shows that $F$ maps into $X^{\odot \odot}$ and sun-reflexivity is only used to identify $X^{\odot \odot}$ with $X$.

Lemma 2.2 makes it possible to prove existence and uniqueness of $\{U(t, s)\}_{(t, s) \in \Delta}$ by successive approximations.

THEOREM 2.3. The variation-of-constants formula

$$
U(t, s) x=T_{0}(t-s) x+\int_{s}^{t} T_{0}^{\odot *}(t-\tau) B(\tau) U(\tau, s) x d \tau, \quad(t, s) \in \Delta, \quad x \in X,
$$

uniquely defines a strongly continuous forward evolutionary system $\{U(t, s)\}_{(t, s) \in \Delta}$, satisfying

$$
\|U(t, s)\| \leqq M e^{[\omega+M K(t, s) \mid(t-s)},
$$

where $M$ and $\omega$ are such that $\left\|T_{0}(t)\right\| \leqq M e^{\omega t}$ and $K(t, s):=\sup _{s \leqq \tau \leqq t}\|B(\tau)\|$. The generation expansion

$$
U(t, s)=\sum_{n=0}^{\infty} U_{n}(t, s)
$$

where $U_{0}(t, s)=T_{0}(t-s), U_{n}(t, s) x=\int_{s}^{t} T_{0}^{\odot *}(t-\tau) B(\tau) U_{n-1}(\tau, s) x d \tau$, converges in the uniform operator topology of $\mathscr{B}(X)$ uniformly on $\Delta$.

The proof employs only standard arguments very similar to those of [19, Proposition 1.2, p. 77] and is therefore omitted. Notice that whenever $t \rightarrow\|B(t)\|$ is measurable we can sharpen the estimate (2.2) and replace it by

$$
\|U(t, s)\| \leqq M \exp \left\{\int_{s}^{t}[\omega+M\|B(\tau)\|] d \tau\right\} .
$$

In the autonomous case the infinitesimal generator of the perturbed system is given by the part $A$ of $A_{0}^{\odot *}+B$ in $X$. We close this section by deriving the non-autonomous analogues of this result both in the forward and backward setting. Let $A(s)$ be the part of $A_{0}^{\odot *}+B(s)$ in $X$, i.e.

$$
\begin{gathered}
A(s) x=A_{0}^{\odot *} x+B(s) x, \\
x \in D(A(s)):=\left\{x \in D\left(A_{0}^{\odot *}\right): A_{0}^{\odot *} x+B(s) x \in X\right\} .
\end{gathered}
$$

LeMmA 2.4. Let $x \in D(A(s))$. Then $(1 /(t-r))(U(t, r) x-x) \rightarrow A(s) x$ as $r, t \rightarrow s$, $r<t$.

Proof. The variation-of-constants formula (2.1) implies that

$$
\frac{1}{t-r}(U(t, r) x-x)=\frac{1}{t-r}\left(T_{0}(t-r) x-x\right)+\frac{1}{t-r} \int_{r}^{t} T_{0}^{\odot *}(t-\tau) B(\tau) U(\tau, r) x d \tau .
$$




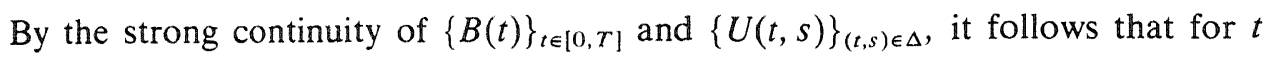
and $r$ tending to $s, r<t$, we have

$$
\begin{aligned}
0= & \lim \left\{\frac{1}{t-r}(U(t, r) x-x)-\frac{1}{t-r}\left(T_{0}(t-r) x-x\right)\right. \\
& \left.-\frac{1}{t-r} \int_{r}^{t} T_{0}^{\odot *}(t-\tau) B(s) x d \tau\right\} \\
= & \lim \left\{\frac{1}{t-r}(U(t, r) x-x)-\frac{1}{t-r} \int_{0}^{t-r} T_{0}^{\odot *}(\tau)\left(A_{0}^{\odot *} x+B(s) x\right) d \tau\right\} .
\end{aligned}
$$

As $A_{0}^{\odot *} x+B(s) x \in X$, the assertion follows.

TheOREM 2.5. Let $x \in D(A(s))$. Then

$$
\begin{gathered}
\left.\frac{\partial^{+}}{\partial t} U(t, s) x\right|_{t=s}=A(s) x, \\
\frac{\partial}{\partial s} U(t, s) x=-U(t, s) A(s) x,
\end{gathered}
$$

where the right derivative in (2.5) and the derivative in (2.6) are in the norm topology of $X$.

Proof. Formula (2.5) immediately follows from Lemma 2.4. In order to show (2.6) let $h>0$. Then

$$
\frac{1}{h}(U(t, s-h)-U(t, s)) x=U(t, s) \frac{1}{h}(U(s, s-h) x-x) \rightarrow U(t, s) A(s) x,
$$

by Lemma 2.4. Further

$$
\begin{aligned}
& \frac{1}{h}(U(t, s)-U(t, s+h)) \\
& =U(t, s+h) \frac{1}{h}(U(s+h, s) x-x) \\
& =U(t, s+h)\left(\frac{1}{h}(U(s+h, s) x-x)-A(s) x\right)+U(t, s+h) A(s) x \\
& \quad \rightarrow U(t, s) A(s) x \text { for } h \downarrow 0,
\end{aligned}
$$

by Lemma 2.4 , the strong continuity of $U(t, s)$ on $\Delta$ and the uniform boundedness of $\|U(t, s+h)\|, 0 \leqq h \leqq 1$.

Theorem 2.5 gives our first answer to the question of the sense in which $A_{0}^{\odot *}+B(s)$ generates the evolutionary system $\{U(t, s)\}_{(t, s) \in \Delta}$. It should be compared with [19, Theorem 5.3.1].

\section{Continuity of the dual system and invariance of $X^{\odot}$}

Recall that $X^{\odot}$ is by definition the largest subspace of $X^{*}$ on which the semigroup $\left\{T_{0}^{*}(t)\right\}_{t \geqq 0}$ is strongly continuous. In [5, Lemma 4.3] we showed that 
in the case of a time-independent perturbation $B: X \rightarrow X^{\odot *}$ the adjoint $\left\{T^{*}(t)\right\}_{t \geqq 0}$ of the perturbed semigroup leaves $X^{\odot}$ invariant and $X^{\odot}$ is the largest subspace on which $\left\{T^{*}(t)\right\}_{t \geqq 0}$ is strongly continuous. However, when the perturbation $B$ depends on time, the situation is not that simple and we have to investigate carefully the relation between various continuity properties of the dual evolutionary system $\left\{U^{*}(s, t)\right\}_{(s, t) \in \Delta^{*}}$ and invariance of $X^{\odot}$. Invariance of $X^{\odot}$ is important, since it enables us to define $\left\{U^{\odot}(s, t)\right\}_{(s, t) \in \Delta^{*}}$ and hence to obtain the extension $\left\{U^{\odot *}(t, s)\right\}_{(t, s) \in \Delta}$ of $\{U(t, s)\}_{(t, s) \in \Delta}$.

LEMMA 3.1. The following conditions are equivalent:

(i) $x^{*} \in X^{\odot}$.

(ii) $\left\|U^{*}(s, t) x^{*}-x^{*}\right\| \rightarrow 0$ as $(t-s) \downarrow 0$ uniformly for $(s, t) \in \Delta^{*}$.

(iii) $\left\|U^{*}(s, t) x^{*}-x^{*}\right\| \rightarrow 0$ as $s \uparrow t$ for all $t \in(0, T]$.

(iv) $\left\|U^{*}(s, t) x^{*}-x^{*}\right\| \rightarrow 0$ as $t \downarrow s$ for some $s \in[0, T)$.

(v) $t \rightarrow U^{*}(s, t) x^{*}$ is continuous $[s, T] \rightarrow X^{*}$ uniformly for $s \in[0, T]$.

Proof. We have

$$
U(t, s)=T_{0}(t-s)+W(t, s)
$$

where

$$
W(t, s) x=\int_{s}^{t} T_{0}^{\odot *}(t-\tau) B(\tau) U(\tau, s) x d \tau .
$$

Since the integrand in (3.2) is bounded, we have $\left\|W(t, s)^{*}\right\|=\|W(t, s)\| \leqq$ $C(t-s)$ for some constant $C<\infty$. Therefore $\left\|W(t, s)^{*}\right\| \rightarrow 0$ as $(t-s) \downarrow 0$ uniformly for $(s, t) \in \Delta^{*}$. The identity

$$
U^{*}(s, t) x^{*}-x^{*}=\left[T_{0}^{*}(t-s) x^{*}-x^{*}\right]+W(t, s)^{*} x^{*}
$$

together with the definition of $X^{\odot}$ now prove that:

$$
\text { (i) } \Rightarrow \text { (ii) } \gg_{(\text {iv })}^{(\text {(iii) }}>_{(i)}^{(\text {i) }}
$$

Let (ii) hold. Then the estimates

$$
\begin{aligned}
& \left\|U^{*}(s, t+h) x^{*}-U^{*}(s, t) x^{*}\right\| \\
& \quad=\left\|U^{*}(s, t) U^{*}(t, t+h) x^{*}-U^{*}(s, t) x^{*}\right\| \\
& \quad \leqq\left\|U^{*}(s, t)\right\|\left\|U^{*}(t, t+h) x^{*}-x^{*}\right\|
\end{aligned}
$$

and

$$
\begin{aligned}
& \left\|U^{*}(s, t-h) x^{*}-U^{*}(s, t) x^{*}\right\| \\
& \quad=\left\|U^{*}(s, t-h) x^{*}-U^{*}(s, t-h) U^{*}(t-h, t) x^{*}\right\| \\
& \quad \leqq\left\|U^{*}(s, t-h)\right\|\left\|x^{*}-U^{*}(t-h, t) x^{*}\right\|
\end{aligned}
$$

and the fact that $\left\|U^{*}(s, t)\right\|$ is uniformly bounded on $\Delta^{*}$ show that (v) holds. Finally (v) trivially implies (iv). 
LEMMA 3.2. The subspace $X^{\odot}$ is invariant under $U^{*}(s, t)$ if and only if $s \rightarrow U^{*}(s, t) x^{\odot}$ is left-continuous from $[0, t]$ to $X^{*}$ for every $x^{\odot} \in X^{\odot}$ and every $t \in(0, T]$.

Proof. This follows immediately from the equivalence (i) $\Leftrightarrow$ (iii) of the preceding lemma and the identity

$$
\begin{aligned}
\left\|U^{*}(s-h, t) x^{\odot}-U^{*}(s, t) x^{\odot}\right\| & \\
& =\left\|U^{*}(s-h, s) U^{*}(s, t) x^{\odot}-U^{*}(s, t) x^{\odot}\right\|, \quad h>0 .
\end{aligned}
$$

The following example shows that $X^{\odot}$ need not be invariant.

EXAmple 3.3. Retarded functional differential equations ( $c f$. [10]).

We consider the retarded functional differential equation (1.6). As the space $X$ we choose $X=C\left([-1,0] ; R^{n}\right)$ of initial functions. $X$ can be identified with $\left\{(\alpha, \phi) \in R^{n} \times C\left([-1,0] ; R^{n}\right): \phi(0)=\alpha\right\} . X^{*}$ is represented by $N B V\left([0,1] ; R^{n^{*}}\right)$, the space of all row-vector-valued functions of bounded variation which vanish on $(-\infty, 0]$, are right-continuous on $(0,1)$ and constant on $[1, \infty)$, with duality pairing

$$
\langle\phi, f\rangle=\int_{0}^{1} d f(\tau) \phi(-\tau), \quad \phi \in X, \quad f \in X^{*} .
$$

The unperturbed equation $\dot{x}=0$, considered as a delay equation, gives rise to a $C_{0}$-semigroup $\left\{T_{0}(t)\right\}_{t \geqq 0}$ on $X$ defined by

$$
\left[T_{0}(t) \phi\right](\theta)=\left\{\begin{array}{ll}
\phi(\theta+t), & \theta+t \leqq 0, \\
\phi(0), & \theta+t>0,
\end{array} \quad \theta \in[-1,0] .\right.
$$

$\left\{T_{0}^{*}(t)\right\}_{t \geqq 0}$ is translation in the other direction and $f \in X^{\odot}$ if and only if it is absolutely continuous on $(0,1)$. It follows that $X^{\odot}$ is the space of all functions $f$ of the form

$$
f(\theta)= \begin{cases}0, & \theta \in(-\infty, 0], \\ c+\int_{0}^{\theta} g(\tau) d \tau, & \theta>0,\end{cases}
$$

for some row vector $c \in R^{n^{*}}$ and $g \in L^{1}\left(R^{+} ; R^{n^{*}}\right)$ with supp $g \subset[0,1]$. Thus $X^{\odot}$ can be represented by $R^{n^{*}} \times L^{1}, L^{1}=\left\{g \in L^{1}\left(R^{+} ; R^{n^{*}}\right)\right.$ : supp $\left.g \subset[0,1]\right\}$, and $X^{\odot *}$ can be represented by $R^{n} \times L^{\infty}\left([-1,0] ; R^{n}\right)$ with pairing

$$
\langle(\alpha, \phi),(c, g)\rangle=c \alpha+\int_{0}^{1} g(\tau) \phi(-\tau) d \tau,
$$

$(\alpha, \phi) \in X^{\odot *},(c, g) \in X^{\odot} .\left\{T_{0}^{\odot}(t)\right\}_{t \geqq 0}$ is given by

$$
T_{0}^{\odot}(t)(c, g)=\left(c+\int_{0}^{t} g(\tau) d \tau, g(t+\cdot)\right),
$$

and $A_{0}^{\odot *}$ by

$$
\begin{aligned}
D\left(A_{0}^{\odot *}\right) & =\{(\alpha, \phi): \phi \text { Lipschitz continuous, } \phi(0)=\alpha\}, \\
A_{0}^{\odot *}(\alpha, \phi) & =\left(0, \phi^{\prime}\right) .
\end{aligned}
$$

For more details, see $[\mathbf{1 0}]$. 
When the perturbation $B(t): X \rightarrow X^{\odot *}$ is defined by

$$
B(t) \phi=(\langle\phi, \zeta(t, \cdot)\rangle, 0)
$$

the problem (1.6) can be written abstractly as

$$
\left.\begin{array}{l}
\frac{d u(t)}{d t}=A_{0}^{\odot *} u(t)+B(t) u(t), \quad t>s, \\
u(s)=\phi .
\end{array}\right\}
$$

A rigorous proof that the evolutionary system $\{U(t, s)\}_{(t, s) \in \Delta}$ corresponding to (3.14) (i.e. given by the variation-of-constants formula $(2.1)$ ) is indeed the evolutionary system corresponding to (1.6) (i.e. $[U(t, s) \phi](\theta)=x(t+\theta ; \phi)$, $\theta \in[-1,0])$ will be given in Section 6 .

For the rest of this example we take $n=1$. Assume that $\zeta$ is given by $\zeta(t, \theta)=H(\theta-t), t \in[0,1]$. Here $H$ is the Heaviside function. Then $B(t) \phi=$ $(\phi(-t), 0)$ so $\{B(t)\}_{t \in[0,1]}$ is strongly continuous. In this case equation (1.6) reduces to

$$
\left.\begin{array}{l}
\dot{x}(t)=x(0), \quad t>s, \\
x(s+\theta)=\phi(\theta), \quad \theta \in[-1,0] .
\end{array}\right\}
$$

The solution $x(t ; \phi)$ of (3.15) is given by

$$
x(t)= \begin{cases}\phi(t-s) & s-1 \leqq t \leqq s \\ \phi(0)+\phi(-s)(t-s), & s \leqq t \leqq 1\end{cases}
$$

and thus

$$
[U(t, s) \phi](\theta)=\left\{\begin{array}{l}
\phi(t-s+\theta), \quad-1 \leqq \theta \leqq-(t-s), \\
\phi(0)+\phi(-s)(t-s+\theta), \quad-(t-s) \leqq \theta \leqq 0 .
\end{array}\right.
$$

Let $f \in X^{\odot}$ have the representation (3.9). Then

$$
\begin{aligned}
\langle U(t, s) \phi, f\rangle & \\
= & c[U(t, s) \phi](0)+\int_{0}^{1} g(\tau)[U(t, s) \phi](-\tau) d \tau \\
= & {\left[c+\int_{0}^{t-s} g(\tau) d \tau\right] \phi(0)+\int_{0}^{t-s}\left[c+\int_{0}^{\tau} g(\theta) d \theta\right] d \tau \phi(-s) } \\
& \quad+\int_{0}^{1-(t-s)} g(t-s+\tau) \phi(-\tau) d \tau,
\end{aligned}
$$

from which it follows that

$$
\begin{aligned}
{\left[U^{*}(s, t) f\right](\theta)=} & {\left[c+\int_{0}^{t-s} g(\tau) d \tau\right] H(\theta) } \\
& +\int_{0}^{t-s}\left[c+\int_{0}^{\tau} g(u) d u\right] d \tau H(\theta-s)+\int_{t-s}^{t-s+\theta} g(\tau) d \tau .
\end{aligned}
$$


We see from (3.19) that unless $f=0$ (i.e. $c=0, g=0) U^{*}(s, t) f$ is discontinuous at $\theta=s$, where it has a jump of magnitude $(t-s) c+\int_{0}^{t-s}(t-s-$ $\tau) g(\tau) d \tau$. Thus $U^{*}(s, t) f$ is not of the form (3.9) and hence it does not belong to $X^{\odot}$. It is also easy to check that $s \rightarrow U^{*}(s, t) f$ is not left-continuous as prescribed by Lemma 3.2.

In Section 5 we shall impose stronger conditions on $B$ in order to obtain invariance of $X^{\odot}$.

\section{Differentiability of orbits}

In Theorem 2.5 we obtained a result on the differentiability of the orbit $t \rightarrow U(t, s) x$ with respect to the norm topology of $X$. Since $X$ is embedded into $X^{\odot *}$, there are at least two other natural topologies in which orbits can be differentiable. We start by looking at weak differentiability.

THEOREM 4.1. For all $x \in X, s \in[0, T)$ and $x^{\odot} \in D\left(A_{0}^{*}\right)$ the real valued function $t \rightarrow\left\langle U(t, s) x, x^{\odot}\right\rangle$ is continuously differentiable with derivative $\left\langle U(t, s) x,\left(A_{0}^{*}+\right.\right.$ $\left.\left.B^{*}(t)\right) x^{\odot}\right\rangle$.

In this theorem we put no restrictions on the initial datum $x$, but only on the test-functionals $x^{\odot}$. Later we shall consider $X$ as a subspace of $X^{\odot *}$ and consider weak*-differentiability of orbits. Since the function $t \rightarrow\left\langle U(t, s) x, x^{\odot}\right\rangle$ must then be differentiable for all functionals $x^{\odot} \in X^{\odot}$, it is clear that extra conditions have to be imposed on $x$.

As with continuity, the fact that $B$ depends on time creates a distinction between "local" properties for $(t-s) \downarrow 0$ and "global" properties of orbits. In particular the invariance of $D\left(A_{0}^{\odot *}\right)$ requires special conditions on $B(t)$.

Proof of Theorem 4.1. If $x^{\odot} \in D\left(A_{0}^{\odot}\right)$, it follows from

$$
\left\langle U(t, s) x, x^{\odot}\right\rangle=\left\langle x, T_{0}^{\odot}(t-s) x^{\odot}\right\rangle+\int_{s}^{t}\left\langle B(\tau) U(\tau, s) x, T_{0}^{\odot}(t-\tau) x^{\odot}\right\rangle d \tau
$$

that

$$
\begin{aligned}
\frac{\partial}{\partial t}\left\langle U(t, s) x, x^{\odot}\right\rangle= & \left\langle x, T_{0}^{\odot}(t-s) A_{0}^{\odot} x^{\odot}\right\rangle \\
& +\left\langle B(t) U(t, s) x, x^{\odot}\right\rangle \\
& +\int_{s}^{t}\left\langle B(\tau) U(\tau, s) x, T_{0}^{\odot}(t-\tau) A_{0}^{\odot} x^{\odot}\right\rangle d \tau \\
= & \left\langle U(t, s) x, A_{0}^{\odot} x^{\odot}\right\rangle+\left\langle B(t) U(t, s) x, x^{\odot}\right\rangle .
\end{aligned}
$$

By the strong continuity of $B$, the right-hand side of (4.2) is a continuous function of $t$.

Now let $x^{\odot} \in D\left(A_{0}^{*}\right)$ and define $x_{n}^{\odot}=n \int_{0}^{1 / n} T_{0}^{\odot}(\tau) x^{\odot} d \tau$. Then $x_{n}^{\odot} \in D\left(A_{0}^{\odot}\right)$ and $\left\|x_{n}^{\odot}-x^{\odot}\right\| \rightarrow 0$ as $n \rightarrow \infty$. We claim that

$$
\frac{\partial}{\partial t}\left\langle U(t, s) x, x_{n}^{\odot}\right\rangle
$$


converges to $\left\langle U(t, s) x, A_{0}^{*} x^{\odot}\right\rangle+\left\langle B(t) U(t, s) x, x^{\odot}\right\rangle$ uniformly for $t \in[s, T]$ as $n \rightarrow \infty$. The assertion then follows from this result by a well-known theorem on termwise differentiation of a sequence (see e.g. [20, Theorem 7.17]).

Since $\|B(t) U(t, s) x\|$ is uniformly bounded, it is clear that $\left\langle B(t) U(t, s) x, x_{n}^{\odot}\right\rangle \rightarrow\left\langle B(t) U(t, s) x, x^{\odot}\right\rangle$ uniformly on $t \in[s, T]$. Next, observe that

$$
f_{n}(t):=\left\langle U(t, s) x, A_{0}^{\odot} x_{n}^{\odot}\right\rangle=\left\langle U(t, s) x, n\left[T_{0}^{\odot}\left(\frac{1}{n}\right) x^{\odot}-x^{\odot}\right]\right\rangle .
$$

converges pointwise to

$$
f(t):=\left\langle U(t, s) x, A_{0}^{*} x^{\odot}\right\rangle, \quad t \in[s, T] .
$$

The estimate

$$
\begin{aligned}
\left|f_{n}\left(t_{1}\right)-f_{n}\left(t_{2}\right)\right| & =\left|\left\langle U\left(t_{1}, s\right) x-U\left(t_{2}, s\right) x, n\left[T_{0}^{\odot}\left(\frac{1}{n}\right) x^{\odot}-x^{\odot}\right]\right\rangle\right| \\
& \leqq\left\|U\left(t_{1}, s\right) x-U\left(t_{2}, s\right) x\right\| n\left\|T_{0}^{\odot}\left(\frac{1}{n}\right) x^{\odot}-x^{\odot}\right\| \\
& \leqq\left\|U\left(t_{1}, s\right) x-U\left(t_{2}, s\right) x\right\| n \sup _{0 \leqq \tau \leqq 1 / n}\left\{\left\|T_{0}(\tau)\right\|\right\}\left\|A_{0}^{*} x^{\odot}\right\| \frac{1}{n}
\end{aligned}
$$

shows that $\left\{f_{n}\right\}$ is equicontinuous. Since a pointwise converging equicontinuous sequence converges uniformly (cf. [11, Theorem 7.5.6]) the proof is complete.

Remark. If $x^{\odot} \in D\left(A_{0}^{*}\right)$, one cannot conclude directly from (4.1) that (4.2) holds with $A_{0}^{\odot}$ replaced by $A_{0}^{*}$. The reason is that the expression $\langle B(\tau) U(\tau, s) x$, $\left.T_{0}^{*}(t-\tau) A_{0}^{*} x^{\odot}\right\rangle$ in the intermediate step is undefined (there is no pairing between $X^{*}$ and $\left.X^{\odot *}\right)$.

We now turn our attention to weak*-differentiability. We start with some lemmas. The first lemma is an analogue of [5, Lemma 4.4] and the proof is omitted.

LeMmA 4.2. For all $x \in X,(1 /(t-s)) \int_{s}^{t} T_{0}^{\odot *}(t-\tau) B(\tau) U(t, s) x d \tau$ converges in the weak ${ }^{*}$-topology of $X^{\odot *}$ to $B(s) x$ as $(t-s) \downarrow 0$ uniformly in $(t, s) \in \Delta$.

LEMMA 4.3. The following conditions are equivalent

(i) $x \in D\left(A_{0}^{\odot *}\right)$.

(ii) $(1 /(t-s))(U(t, s) x-x)$ converges in the weak ${ }^{*}$-topology of $X^{\odot *}$ as $(t-s) \downarrow 0$, uniformly for $(t, s) \in \Delta$.

(iii) $(1 /(t-s))(U(t, s) x-x)$ converges in the weak ${ }^{*}$-topology of $X^{\odot *}$ as $t \downarrow s$ for all $s \in[0, T)$.

(iv) There exists an $s \in[0, T)$ such that $(1 /(t-s))(U(t, s) x-x)$ converges in the weak ${ }^{*}$-topology of $X^{\odot *}$ as $t \downarrow s$.

When the mutually equivalent conditions (i)-(iv) are satisfied, the limit is $\left(A_{0}^{\odot *}+B(s)\right) x$.

The proof, which is based on Lemma 4.2 and the variation-of-constants formula (2.1) is straightforward and therefore omitted. 
Definition 4.4. A function $f:[a, b] \rightarrow X^{\odot *}$ is weak*-differentiable [from the right] with [right-] weak*-derivative $g$ if for every $x^{\odot} \in X^{\odot}$, the real valued function $t \rightarrow\left\langle f(t), x^{\odot}\right\rangle$ is differentiable [from the right] with [right-] derivative $\left\langle g(t), x^{\odot}\right\rangle$. A weak*-differentiable function $f$ is continuously weak ${ }^{*}$-differentiable if in addition the function $t \rightarrow\left\langle g(t), x^{\odot}\right\rangle$ is continuous for all $x^{\odot} \in X^{\odot}$.

LEMMA 4.5. The following conditions are equivalent.

(i) $U(t, s) D\left(A_{0}^{\odot *}\right) \subset D\left(A_{0}^{\odot *}\right) \forall(t, s) \in \Delta$.

(ii) $t \rightarrow U(t, s) x$ considered as a function $[s, T] \rightarrow X^{\odot *}$ is weak ${ }^{*}$-differentiable from the right for all $s \in[0, T)$ and all $x \in D\left(A_{0}^{\odot *}\right)$.

When the equivalent conditions (i) and (ii) are satisfied, the right-weak ${ }^{*}$-derivative of $U(t, s) x$ equals $\left(A_{0}^{\odot *}+B(t)\right) U(t, s) x$.

Proof. Since $(1 / h)\{U(t+h, s) x-U(t, s) x\}=(1 / h)\{U(t+h, t) U(t, s) x-U(t, s) x\}$, the assertion follows from the equivalence (i) $\Leftrightarrow$ (iii) of Lemma 4.3.

THEOREM 4.6. The following conditions are equivalent:

(i) $U(t, s) D\left(A_{0}^{\odot *}\right) \subset D\left(A_{0}^{\odot *}\right) \forall(t, s) \in \Delta$ and for all $x \in D\left(A_{0}^{\odot *}\right), s \in[0, T)$

$$
\sup _{s \leqq t \leqq T}\left\|A_{0}^{\odot *} U(t, s) x\right\|<\infty .
$$

(ii) For every $x \in D\left(A_{0}^{\odot *}\right)$ and $s \in[0, T)$ the function $t \rightarrow U(t, s) x$ from $[s, T]$ into $X^{\odot *}$ is continuously weak ${ }^{*}$-differentiable.

When the equivalent conditions (i) and (ii) hold, the weak ${ }^{*}$-derivative of $U(t, s) x$ equals $\left(A_{0}^{\odot *}+B(t)\right) U(t, s) x$.

Proof. By Lemma 4.5 the first part of (i) implies that $\partial^{+} / \partial t\left\langle U(t, s) x, x^{\odot}\right\rangle=$ $\left\langle\left(A_{0}^{\odot *}+B(t)\right) U(t, s) x, x^{\odot}\right\rangle \forall x^{\odot} \in X^{\odot}$. To prove that (ii) holds it suffices to show that the right derivative is continuous. If $x^{\odot} \in D\left(A_{0}^{\odot}\right)$, then $\partial^{+} / \partial t\left\langle U(t, s) x, x^{\odot}\right\rangle=\left\langle U(t, s) x, A_{0}^{\odot} x^{\odot}\right\rangle+\left\langle B(t) U(t, s) x, x^{\odot}\right\rangle$ is indeed continuous in $t$. If $x^{\odot} \in X^{\odot}, x_{n}^{\odot} \in D\left(A_{0}^{\odot}\right),\left\|x_{n}^{\odot}-x^{\odot}\right\| \rightarrow 0$, then the latter part of (i) shows that $\left\langle\left(A_{0}^{\odot *}+B(t)\right) U(t, s) x, x_{n}^{\odot}\right\rangle \rightarrow\left\langle\left(A_{0}^{\odot *}+B(t)\right) U(t, s) x, x^{\odot}\right\rangle$ uniformly in $t \in[s, T]$, and hence the limit is continuous in $t \in[s, T]$.

Conversely, if (ii) holds, then by Lemma $4.5 U(t, s) D\left(A_{0}^{\odot *}\right) \subset D\left(A_{0}^{\odot *}\right) \forall(t, s) \epsilon$ $\Delta$ and $\partial / \partial t\left\langle U(t, s) x, x^{\odot}\right\rangle=\left\langle\left(A_{0}^{\odot *}+B(t)\right) U(t, s) x, x^{\odot}\right\rangle$. Since the derivative is now continuous on $[s, T]$ we have

$$
\sup _{s \leqq t \leqq T}\left|\left\langle\left(A_{0}^{\odot *}+B(t)\right) U(t, s) x, x^{\odot}\right\rangle\right|<\infty
$$

for all $x^{\odot} \in X^{\odot}$ and hence the latter condition in (i) holds by the uniform boundedness principle.

It is often possible to verify that condition (i) of Theorem 4.6 holds by exploiting special properties of the range of $B(t)$ or additional smoothness of $B(t)$.

Hypothesis 4.7. A subspace $Z$ of $X^{\odot *}$ is said to satisfy the hypothesis if for every $s \in[0, T)$ and every continuous function $h:[s, T] \rightarrow X^{\odot *}$ the range of which 
is contained in $Z$, we have

(i) $\int_{s}^{t} T_{0}^{\odot *}(t-\tau) h(\tau) d \tau \in D\left(A_{0}^{\odot *}\right), s \leqq t \leqq T$,

(ii) $\left\|A_{0}^{\odot *} \int_{s}^{t} T_{0}^{\odot *}(t-\tau) h(\tau) d \tau\right\| \leqq M \sup _{s \leqq r \leqq t}\|h(\tau)\|, s \leqq t \leqq T$.

These conditions are inspired by and should be compared with those of $[7, \mathrm{p}$. 330-331], see also [8, 9].

THEOREM 4.8. If the range of $B(t)$, for all $t \in[0, T]$, is contained in a subspace $Z$ satisfying Hypothesis 4.7 , then $U(t, s)$ satisfies the mutually equivalent conditions in Theorem 4.6.

Proof. Let $x \in D\left(A_{0}^{\odot *}\right)$. Since $T_{0}(t)$ leaves $D\left(A_{0}^{\odot *}\right)$ invariant, the first term to the right of the variation-of-constants formula (2.1) belongs to $D\left(A_{0}^{\odot *}\right)$ and since $\tau \rightarrow B(\tau) U(\tau, s) x$ is continuous from $[s, T]$ into $X^{\odot *}$, condition (i) above guarantees that the latter term also belongs to $D\left(A_{0}^{\odot *}\right)$.

From (2.1) it follows that

$$
A_{0}^{\odot *} U(t, s) x=T_{0}^{\odot *}(t-s) A_{0}^{\odot *} x+A_{0}^{\odot *} \int_{s}^{t} T_{0}^{\odot *}(t-\tau) B(\tau) U(\tau, s) x d \tau,
$$

so, by (ii) above,

$$
\left\|A_{0}^{\odot *} U(t, s) x\right\| \leqq M e^{\omega(t-s)}\left\|A_{0}^{\odot *} x\right\|+M \sup _{s \leqq \tau \leqq t}\|B(\tau) U(\tau, s) x\|,
$$

and hence condition (i) of Theorem 4.6 holds.

THEOREM 4.9. If $t \rightarrow B(t)$ is Lipschitz continuous from $[0, T]$ to $\mathscr{B}\left(X, X^{\odot *}\right)$, then the mutually equivalent conditions of Theorem 4.6 hold.

Proof. The proof is based on the characterisation of $D\left(A_{0}^{\odot *}\right)$ as the Favard class of $\left\{T_{0}(t)\right\}_{t \geqq 0}$ (cf. [4, Corollary 2.1.5] and [6, Section 3.4]): $x \in D\left(A_{0}^{\odot *}\right)$ if and only if $\left\|T_{0}(h) x-x\right\|=O(h)$ as $h \downarrow 0$.

We first show that if $x \in D\left(A_{0}^{\odot *}\right)$; then there is a constant $L<\infty$ depending only on $x$ such that

$$
\|U(t+h, s) x-U(t, s) x\| \leqq L h, \quad(t, s) \in \Delta, \quad h>0 .
$$

Using the variation-of-constants formula (2.1) one finds

$$
\begin{aligned}
U(t+h, s) x-U(t, s) x= & T_{0}(t-s)\left[T_{0}(h) x-x\right] \\
& +\int_{s-h}^{s} T_{0}^{\odot *}(t-\tau) B(\tau+h) U(\tau+h, s) x d \tau \\
& +\int_{s}^{t} T_{0}^{\odot *}(t-\tau)[B(\tau+h)-B(\tau)] U(\tau+h, s) x d \tau \\
& +\int_{s}^{t} T_{0}^{\odot *}(t-\tau) B(\tau)[U(\tau+h, s) x-U(\tau, s) x] d \tau
\end{aligned}
$$

Since $t \rightarrow B(t)$ and $h \rightarrow T_{0}(h) x$ are Lipschitz continuous and $\left\|T_{0}(t)\right\|$ and $\|U(t, s)\|$ are bounded on $[0, T]$ and $\Delta$, respectively, (4.5) follows from Gronwall's Lemma.

Fix $(t, s) \in \Delta, x \in D\left(A_{0}^{\odot *}\right)$. Applying $T_{0}(h)-I$ to both sides of the variation-of- 
constants formula (2.1) one obtains

$$
\begin{aligned}
& T_{0}(h) U(t, s) x-U(t, s) x \\
& =T_{0}(t-s)\left[T_{0}(h) x-x\right]+\int_{s-h}^{s} T_{0}^{\odot *}(t-\tau) B(\tau+h) U(\tau+h, s) x d \tau \\
& \quad+\int_{s}^{t-h} T_{0}^{\odot *}(t-\tau)\{B(\tau+h) U(\tau+h, s) x-B(\tau) U(\tau, s) x\} d \tau \\
& \quad-\int_{t-h}^{t} T_{0}^{\odot *}(t-\tau) B(\tau) U(\tau, s) x d \tau .
\end{aligned}
$$

Using the Lipschitz continuity of $B,(4.5)$ and the fact that $x$ is in the Favard class of $\left\{T_{0}(t)\right\}_{t \geqq 0}$, one finds that

$$
\left\|T_{0}(h) U(t, s) x-U(t, s) x\right\| \leqq L^{\prime} h \quad(t, s) \in \Delta, \quad h>0
$$

for some finite constant $L^{\prime}$ depending only on $x$. In particular $U(t, s) x \in D\left(A_{0}^{\odot *}\right)$. Moreover, since by definition $A_{0}^{\odot *} U(t, s) x$ equals the weak*-limit of $(1 / h)\left[T_{0}^{\odot *}(h) U(t, s) x-U(t, s) x\right]$ as $h \downarrow 0$, it follows from a corollary of the uniform boundedness principle that

$$
\begin{aligned}
\left\|A_{0}^{\odot *} U(t, s) x\right\| & \leqq \limsup _{h \downarrow 0}\left\|\frac{1}{h}\left[T_{0}^{\odot *}(h) U(t, s) x-U(t, s) x\right]\right\| \\
& \leqq L^{\prime} .
\end{aligned}
$$

Thus condition (i) of Theorem 4.6 holds.

We illustrate the use of the preceding theorems by a couple of examples.

\section{EXAMPLE 4.10. Retarded functional differential equations.}

We consider the retarded functional differential equation (1.6) as a perturbation problem as explained in Example 3.3. For the sake of simplicity we take $n=1$. We assume that $t \rightarrow \zeta(t, \cdot)$ is a weak ${ }^{*}$-continuous function $[0, T] \rightarrow$ $N B V[0,1]$. Then $\{B(t)\}_{t \in[0, T]}$ defined by (3.13) is strongly continuous.

Let $Z$ be the one-dimensional subspace $\{(\alpha, 0): \alpha \in R\}$ of $X^{\odot *}$. Then the range of $B(t)$ is contained in $Z$. We verify conditions (i) and (ii) of Hypothesis 4.7. Let $h:[s, T] \rightarrow X^{\odot *}$ be continuous with $h(t) \in Z, t \in[s, T], h(t)=(\alpha(t), 0), \alpha$ a continuous scalar function and let $(c, g) \in X^{\odot}$. Then

$$
\begin{aligned}
& \left\langle\int_{s}^{t} T_{0}^{\odot *}(t-\tau) h(\tau) d \tau,(c, g)\right\rangle \\
& \quad=\int_{s}^{t}\left\langle h(\tau), T_{0}^{\odot}(t-\tau)(c, g)\right\rangle d \tau \\
& \quad=\int_{s}^{t}\left\langle(\alpha(\tau), 0),\left(c+\int_{0}^{t-\tau} g(\theta) d \theta, g(t-\tau+\cdot)\right)\right\rangle d \tau \\
& \quad=\int_{s}^{t}\left[c \alpha(\tau)+\alpha(\tau) \int_{0}^{t-\tau} g(\theta) d \theta\right] d \tau
\end{aligned}
$$




$$
\begin{aligned}
& =c \int_{s}^{t} \alpha(\tau) d \tau+\int_{0}^{t-s} g(\theta) \int_{s}^{t-\theta} \alpha(\tau) d \tau d \theta \\
& =c \int_{s}^{t} \alpha(\tau) d \tau+\int_{0}^{1} g(\theta) \psi(-\theta) d \theta \\
& =\left\langle\left(\int_{s}^{t} \alpha(\tau) d \tau, \psi\right),(c, g)\right\rangle,
\end{aligned}
$$

where

$$
\psi(\theta)= \begin{cases}\int_{s}^{t+\theta} \alpha(\tau) d t, & -\min \{1, t-s\} \leqq \theta \leqq 0 \\ 0, & -1 \leqq \theta \leqq-\min \{1, t-s\}\end{cases}
$$

It follows that

$$
\int_{s}^{t} T_{0}^{\odot *}(t-\tau) h(\tau) d \tau=\left(\int_{s}^{t} \alpha(\tau) d \tau, \psi\right),
$$

and since $\psi$ is Lipschitz continuous on $[-1,0]$ and

$$
\psi(0)=\int_{s}^{t} \alpha(\tau) d \tau, \quad \int_{s}^{t} T_{0}^{\odot *}(t-\tau) h(\tau) d \tau \in D\left(A_{0}^{\odot *}\right) .
$$

Moreover

$$
A_{0}^{\odot *} \int_{s}^{t} T_{0}^{\odot *}(t-\tau) h(\tau) d \tau=\left(0, \psi^{\prime}\right)
$$

where

$$
\psi^{\prime}(\theta)= \begin{cases}\alpha(t+\theta), & -\min \{1, t-s\} \leqq \theta \leqq 0 \\ 0, & -1 \leqq \theta \leqq-\min \{1, t-s)\}\end{cases}
$$

Since $\alpha$ is continuous, the supremum of $\psi^{\prime}(\theta)$ over $t \in[s, T]$ is obviously finite. Thus $Z$ satisfies Hypothesis 4.7 and Theorem 4.8 can be applied.

Example 4.11. Age-dependent population dynamics.

We consider the following dual formulation of the age-dependent population problem:

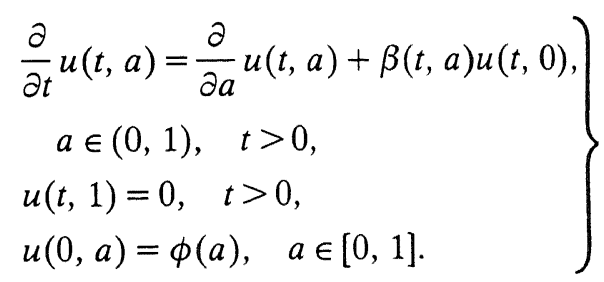

This problem, with $\beta$ independent of $t$, was treated in the context of perturbations of dual semigroups in detail in [5]. The unperturbed semigroup $\left\{T_{0}(t)\right\}_{t \geqq 0}$ corresponding to the equation $\partial u / \partial t=\partial u / \partial a$ is translation to the left acting on $X=C_{0}[0,1)=\left\{\phi \in C[0,1): \lim _{a \uparrow 1} \phi(a)=0\right\}$. Then $X^{*}=M[0,1)$, the Banach space of all Borel measures on $[0,1), X^{\odot}$ is the subspace of all absolutely continuous measures identified with $L^{1}[0,1]$, and $X^{\odot *}=L^{\infty}[0,1]$. The unper- 
turbed generator on $X^{\odot *}$ is given by $D\left(A_{0}^{\odot *}\right)=\left\{\phi \in C_{0}[0,1): \phi\right.$ Lipschitz continuous $\}, A_{0}^{\odot *} \phi=\phi^{\prime} .\left\{T_{0}^{\odot *}(t)\right\}_{t \geqq 0}$ is translation to the left.

When the fecundity function $\beta(t, a)$ depends on both age and time the perturbation $B(t): X \rightarrow X^{\odot *}$ is time-dependent and given by $B(t) \phi=\phi(0) \beta(t, \cdot)$. It is immediately seen that if $\beta$ is Lipschitz continuous in $t$, with Lipschitz constant independent of $a \in[0,1]$, then $t \rightarrow B(t)$ is Lipschitz continuous from $[0, T]$ to $\mathscr{B}\left(X, X^{\odot *}\right)$ and hence by Theorem 4.9 the conditions of Theorem 4.6 hold. On the other hand, if $\beta$ is Lipschitz continuous in $a$, uniformly for $t \in[0, T]$, condition (i) of Theorem 4.6 can easily be verified directly. We therefore conclude that in order to have $(\partial / \partial t) U(t, s) x=\left(A_{0}^{\odot *}+B(t)\right) U(t, s) x$ in the weak*-sense for $x \in D\left(A_{0}^{\odot *}\right)$ it is sufficient that the fecundity function $\beta$ is Lipschitz continuous in either the time or age variable, uniformly in the other variable.

We close this section by noting that the differentiability results can be reformulated in terms of solutions to the Cauchy problem

$$
\left.\begin{array}{l}
\frac{d}{d t} u(t)=\left(A_{0}^{\odot *}+B(t)\right) u(t), \quad s<t \leqq T, \\
u(s)=x^{\odot *},
\end{array}\right\}
$$

in an appropriate weak or weak*-sense.

In order to be a weak solution to (4.10), $u$ must take values in $X$ and $t \rightarrow\left\langle u(t), x^{\odot}\right\rangle$ must be differentiable for every $x^{\odot} \in D\left(A_{0}^{*}\right)$. Weak ${ }^{*}$-solutions are in principle $X^{\odot *}$-valued functions, which are weak*-differentiable. Hence the class of test-functionals is larger in the weak*-case. The price one has to pay for this is that the class of initial data has to be smaller and, in particular, the solutions will actually take values in $D\left(A_{0}^{\odot *}\right)$. We give a formal definition.

Definition 4.12. (a) A function $u:$ [s, $T] \rightarrow X$ is called a weak solution (cf. [2]) to the Cauchy problem (4.10) with $x^{\odot *}=x \in X$ if $u(s)=x$ and if for every $x^{\odot} \in D\left(A_{0}^{*}\right)$ the real-valued function $t \rightarrow\left\langle u(t), x^{\odot}\right\rangle$ is continuously differentiable and

$$
\frac{d}{d t}\left\langle u(t), x^{\odot}\right\rangle=\left\langle u(t),\left(A_{0}^{*}+B^{*}(t)\right) x^{\odot}\right\rangle
$$

for $s<t \leqq T$.

(b) A function $u:[s, T] \rightarrow X^{\odot *}$ is called a weak ${ }^{*}$-solution to $(4.10)$ if $u(s)=$ $x^{\odot *}, u(t) \in D\left(A_{0}^{\odot *}\right), s<t \leqq T$ and $u$ is continuously weak ${ }^{*}$-differentiable on $(s, T]$ with weak*-derivative $\left(A_{0}^{\odot *}+B(\cdot)\right) u(\cdot)$.

THEOREM 4.13. For all $x \in X, s \in[0, T), t \rightarrow U(t, s) x$ is the unique weak solution to the forward problem (4.10).

Proof. That $t \rightarrow U(t, s) x$ is a weak solution is just Theorem 4.1.

To prove uniqueness, let $u$ be an arbitrary weak solution to (4.10) and let 
$x^{\odot} \in D\left(A_{0}^{*}\right)$. Then $T_{0}^{\odot}(t-\tau) x^{\odot} \in D\left(A_{0}^{*}\right)$ and hence

$$
\begin{aligned}
\frac{d}{d t} & \left\langle T_{0}(t-\tau) u(\tau), x^{\odot}\right\rangle \\
& =\frac{d}{d t}\left\langle u(\tau), T_{0}^{\odot}(t-\tau) x^{\odot}\right\rangle \\
& =\left\langle u(\tau),\left(A_{0}^{*}+B^{*}(\tau)\right) T_{0}^{\odot}(t-\tau) x^{\odot}\right\rangle-\left\langle u(\tau), A_{0}^{*} T_{0}^{\odot}(t-\tau) x^{\odot}\right\rangle \\
& =\left\langle u(\tau), B^{*}(\tau) T_{0}^{\odot}(t-\tau) x^{\odot}\right\rangle \\
& =\left\langle T_{0}^{\odot *}(t-\tau) B(\tau) u(\tau), x^{\odot}\right\rangle .
\end{aligned}
$$

By integrating both sides of (4.12) from $s$ to $t$ one obtains

$$
\left\langle u(t), x^{\odot}\right\rangle-\left\langle T_{0}(t-s) u(s), x^{\odot}\right\rangle=\int_{s}^{t}\left\langle T_{0}^{\odot *}(t-\tau) B(\tau) u(\tau), x^{\odot}\right\rangle d \tau .
$$

Since $D\left(A_{0}^{*}\right)$ is weak*-dense in $X^{*}$, (4.13) implies that

$$
u(t)=T_{0}(t-s) u(s)+\int_{s}^{t} T_{0}^{\odot *}(t-\tau) B(\tau) u(\tau) d \tau
$$

and therefore $u(t)=U(t, s) x$ by Theorem 2.3.

THEOREM 4.14. Let condition (i) in Theorem 4.6 hold. Then for every $x \in D\left(A_{0}^{\odot *}\right), s \in[0, T)$, the function $t \rightarrow U(t, s) x$ is the unique weak ${ }^{*}$-solution of the forward problem (4.10).

Proof. That $t \rightarrow U(t, s) x$ is a weak ${ }^{*}$-solution of (4.10) is the content of Theorem 4.6. The uniqueness proof is very similar to that of Theorem 4.13 and therefore omitted.

\section{Going full circle}

In the autonomous case, where $X^{\odot}$ is always invariant under the perturbed dual semigroup, one can define by duality a semigroup on $X^{\odot *}$ which is an extension of the semigroup given by the variation-of-constants formula on $X$. In this sense the circle of the four spaces $X, X^{*}, X^{\odot}, X^{\odot *}$ and the corresponding semigroups is closed. As Example 3.3 shows, strong continuity of the timedependent perturbation $B(t)$ is not enough to guarantee invariance of $X^{\odot}$ under the dual evolutionary system. From now on we shall therefore make the stronger assumption:

Assumption 5.1. The mapping $t \rightarrow B(t)$ is continuous from $[0, T]$ to $\mathscr{B}\left(X, X^{\odot *}\right)$ equipped with the operator norm.

If we make the slightly weaker assumption that both $B(t)$ and $B^{*}(t)$ are strongly continuous, we can define $U^{\odot}(s, t)$ directly by solving a variation-ofconstants formula "at the right-hand side" (i.e. with $X^{\odot}$ as the small space embedded into the big space $\left.X^{*}\right)$. However, we have not been able to show that under merely this assumption necessarily $U^{\odot}(s, t)$ is the restriction of $U^{*}(s, t)$ to 
$X^{\odot}$. Moreover, in our main applications the range of $B(t)$ is a fixed finite dimensional subspace of $X^{\odot *}$ and strong continuity of both $B(t)$ and $B^{*}(t)$ implies Assumption 5.1 so the possible loss of generality is only minor.

The first lemma shows that Assumption 5.1 guarantees that $U(t, s)$ (and consequently $U^{*}(s, t)$ as well) is continuous along lines $t-s=$ constant in $\Delta$ (resp. $\Delta^{*}$ ) with respect to the operator norm.

LEMMA 5.2. The norm $\|U(t+h, s+h)-U(t, s)\| \rightarrow 0$ as $h \rightarrow 0$ uniformly for $(t, s) \in \Delta$.

Proof. Using the variation-of-constants formula we have, independently of the sign of $h, \quad[U(t+h, s+h) x-U(t, s) x]$

$$
\begin{aligned}
= & \int_{s}^{t} T_{0}^{\odot *}(t-\tau)[B(\tau+h)-B(\tau)] U(\tau+h, s+h) x d \tau \\
& +\int_{s}^{t} T_{0}^{\odot *}(t-\tau) B(\tau)[U(\tau+h, s+h) x-U(\tau, s) x] d \tau .
\end{aligned}
$$

There is a constant $M<\infty$ such that

$$
\begin{aligned}
\|U(t+h, s+h) x-U(t, s) x\| \leqq & M\|x\| \sup _{0 \leqq \tau \leqq T}\|B(\tau+h)-B(\tau)\| \\
& +M \int_{s}^{t}\|U(\tau+h, s+h) x-U(\tau, s) x\| d \tau .
\end{aligned}
$$

The assertion now follows from Gronwall's Lemma.

THEOREM 5.3. The subspace $X^{\odot}$ is invariant under $U^{*}(s, t)$.

Proof. By Lemma 3.2 we have to show that $s \rightarrow U^{*}(s, t) x^{\odot}$ is left-continuous for all $t \in(0, T]$ and all $x^{\odot} \in X^{\odot}$. But this follows from the fact that

$$
U^{*}(s-h, t) x^{\odot}=U^{*}(s-h, t-h) U^{*}(t-h, t) x^{\odot},
$$

since $U^{*}(t-h, t) x^{\odot} \rightarrow x^{\odot}$ by (i) $\Rightarrow$ (iii) of Lemma 3.1 and $U^{*}(s-h, t-h) \rightarrow U^{*}(s, t)$ in norm by Lemma 5.2 .

Since $X^{\odot}$ is invariant under $U^{*}(s, t),(s, t) \in \Delta^{*}, U^{\odot}(s, t):=\left.U^{*}(s, t)\right|_{X^{\odot}}$ is a backward evolutionary system on $X^{\odot}$ and $U^{\odot *}(t, s)$ is a forward system on $X^{\odot *}$, which extends $U(t, s)$. By Lemma 3.1 and Lemma 3.2 we know that $U^{\odot}(s, t) x^{\odot}$ is continuous in $t \in[s, T]$, uniformly for $s \in[0, T]$ and left-continuous in $s \in[0, t]$ for all $t \in[0, T]$. Our next result states that $U^{\odot}(s, t) x^{\odot}$ is actually jointly continuous.

TheOREM 5.4. The system $\left\{U^{\odot}(s, t)\right\}_{(s, t) \in \Delta^{*}}$ is a strongly continuous backward evolutionary system.

To prove Theorem 5.4 one only has to show that $(s, t) \rightarrow U^{\odot}(s, t) x^{\odot}$ is continuous from $\Delta^{*}$ to $X^{\odot}$ for all $x^{\odot} \in X^{\odot}$. But this is a straightforward estimation of $\left\|U^{\odot}(s, t) x^{\odot}-U^{\odot}\left(s_{0}, t_{0}\right) x^{\odot}\right\|$ using Lemma 3.1, Lemma 3.2 and Lemma 5.2.

We now state a result, which tells us in what sense $\{U(t, s)\}_{(t, s) \in \Delta}$ is a solution to the backward problem (1.5). Later (Theorem 5.8) we shall reformulate the result as a statement about $\left\{U^{\odot}(s, t)\right\}_{(s, t) \in \Delta^{*}}$. 
THEOREM 5.5. The function $s \rightarrow U^{\odot *}(t, s) x^{\odot *}$ is continuously weak ${ }^{*}$ differentiable on $[0, t]$ if and only if $x^{\odot *}=x \in D\left(A_{0}^{\odot *}\right)$ and in that case the derivative equals $-U^{\odot *}(t, s)\left(A_{0}^{\odot *}+B(s)\right) x$.

Proof. Let us first consider $x \in X$. Then

$$
\frac{1}{h}\left\langle U^{\odot *}(t, s-h) x-U^{\odot *}(t, s) x, x^{\odot}\right\rangle=\frac{1}{h}\left\langle U(s, s-h) x-x, U^{\odot}(s, t) x^{\odot}\right\rangle .
$$

By Lemma 4.3, the right-hand side converges as $h \downarrow 0$ if and only if $x \in D\left(A_{0}^{\odot *}\right)$ and the limit is then $\left\langle\left(A_{0}^{\odot *}+B(s)\right) x, U^{\odot}(s, t) x^{\odot}\right\rangle$. Thus

$$
\frac{\partial^{-}}{\partial s}\left\langle U(t, s) x, x^{\odot}\right\rangle=-\left\langle\left(A_{0}^{\odot *}+B(s)\right) x, U^{\odot}(s, t) x^{\odot}\right\rangle
$$

if and only if $x \in D\left(A_{0}^{\odot *}\right)$. By Theorem $5.4 U^{\odot}(s, t) x^{\odot}$ is continuous and hence the left-derivative is continuous, implying that $\left\langle U(t, s) x, x^{\odot}\right\rangle$ is continuously differentiable. The lemma has now been proved for the case $x \in X$.

Assume that $s \rightarrow U^{\odot *}(t, s) x^{\odot *}$ is continuously weak ${ }^{*}$-differentiable. Take $x^{\odot} \in X^{\odot}$. Then $(1 / h)\left\langle U^{\odot *}(t, t-h) x^{\odot *}-x^{\odot *}, x^{\odot}\right\rangle$ converges as $h \downarrow 0$, in particular it is bounded. By the uniform boundedness principle $(1 / h) \| U^{\odot *}(t, t-h) x^{\odot *}-$ $x^{\odot *} \| \leqq M$ for some $M<\infty$ and $h$ sufficiently small. Lemma 3.1 (with $U^{*}$ replaced by $U^{\odot *}$ and $X^{\odot}$ by $X^{\odot \odot}$ ) then implies that $x^{\odot *} \in X^{\odot \odot}=X$.

In the autonomous case we derived an alternative variation-of-constants formula for the semigroup on $X$ with the roles of the perturbed and unperturbed semigroups interchanged [5, Section 4]. The proof was based on the unique correspondence between a $C_{0}$-semigroup and its generator. In the next proposition we derive by a more direct proof a similar formula for $U(t, s)$ and by duality we obtain a variation-of-constants formula for $U^{\odot}(s, t)$.

THEOREM 5.6. The following variation-of-constants formulae hold.

$$
\begin{gathered}
U(t, s) x=T_{0}(t-s) x+\int_{s}^{t} U^{\odot *}(t, \sigma) B(\sigma) T_{0}(\sigma-s) x d \sigma, \quad x \in X, \\
U^{\odot}(s, t) x^{\odot}=T_{0}^{\odot}(t-s) x^{\odot}+\int_{s}^{t} T_{0}^{*}(\sigma-s) B^{*}(\sigma) U^{\odot}(\sigma, t) x^{\odot} d \sigma, \quad x^{\odot} \in X^{\odot}, \\
U^{\odot}(s, t) x^{\odot}=T_{0}^{\odot}(t-s) x^{\odot}+\int_{s}^{t} U^{*}(s, \tau) B^{*}(\tau) T_{0}^{\odot}(t-\tau) x^{\odot} d \tau, \quad x^{\odot} \in X^{\odot} .
\end{gathered}
$$

Proof. Let $x^{\odot} \in X^{\odot}, x \in D\left(A_{0}\right)$. By Theorem 5.5 we obtain

$$
\begin{aligned}
& \frac{\partial}{\partial \sigma}\left\langle U(t, \sigma) T_{0}(\sigma-s) x, x^{\odot}\right\rangle \\
&=\left\langle U(t, \sigma) A_{0} T_{0}(\sigma-s) x, x^{\odot}\right\rangle \\
&-\left\langle U^{\odot *}(t, \sigma) A_{0}^{\odot *} T_{0}(\sigma-s) x, x^{\odot}\right\rangle \\
&-\left\langle U^{\odot *}(t, \sigma) B(\sigma) T_{0}(\sigma-s) x, x^{\odot}\right\rangle \\
&=-\left\langle U^{\odot *}(t, \sigma) B(\sigma) T_{0}(\sigma-s) x, x^{\odot}\right\rangle .
\end{aligned}
$$


It follows from (5.6) that

$$
\begin{aligned}
& \left\langle\int_{s}^{t} U^{\odot *}(t, \sigma) B(\sigma) T_{0}(\sigma-s) x d \sigma, x^{\odot}\right\rangle \\
& \quad=\int_{s}^{t}\left\langle U^{\odot *}(t, \sigma) B(\sigma) T_{0}(\sigma-s) x, x^{\odot}\right\rangle d \sigma \\
& \quad=-\int_{s}^{t} \frac{\partial}{\partial s}\left\langle U(t, \sigma) T_{0}(\sigma-s) x, x^{\odot}\right\rangle d \sigma \\
& \quad=-\left\langle T_{0}(t-s) x, x^{\odot}\right\rangle+\left\langle U(t, s) x, x^{\odot}\right\rangle .
\end{aligned}
$$

Since $D\left(A_{0}\right)$ is dense in $X$ and both sides of (5.7) are continuous in $x$ (also if $x \in X)$ it follows that (5.3) holds.

We obtain (5.4) from (5.3) by duality and (5.5) from the original variation-ofconstants formula (2.1) for $U(t, s)$.

With the aid of the variation-of-constants formula (5.4) analogues of Theorem 4.13 and Theorem 5.5 can be proved.

THEOREM 5.7. The function $t \rightarrow U^{*}(s, t) x^{*}$ is continuously weak ${ }^{*}$-differentiable in $t \in[s, T]$ if and only if $x^{*}=x^{\odot} \in D\left(A_{0}^{*}\right)$ and in that case the derivative equals $U^{*}(s, t)\left(A_{0}^{*}+B^{*}(t)\right) x^{\odot}$.

THEOREM 5.8. For all $x^{\odot} \in X^{\odot}, t \in(0, T], s \rightarrow U^{\odot}(s, t) x^{\odot}$ is the unique weak solution to the backward problem

$$
\left.\begin{array}{l}
\frac{d}{d s} u(s)=-\left(A_{0}^{\odot}+B^{*}(s)\right) u(s), \quad 0 \leqq s<t \\
u(t)=x^{\odot} \in X^{\odot}
\end{array}\right\}
$$

on $X^{\odot}$.

Except for uniqueness this is simply a reformulation of Theorem 5.5. Uniqueness is proved as in Theorem 4.14.

For the sake of completeness we close this section by stating the analogue of Theorem 4.14 on $X^{\odot}$.

THEOREM 5.9. Suppose $U^{\odot}(s, t) D\left(A_{0}^{*}\right) \subset D\left(A_{0}^{*}\right)$ for all $(s, t) \in \Delta^{*}$ and $\sup _{0 \leq s \leq t}\left\|A_{0}^{*} U^{\odot}(s, t) x^{\odot}\right\|<\infty$ for all $x^{\odot} \in D\left(A_{0}^{*}\right)$ and all $t \in(0, T]$. Then for every $x^{\odot} \in D\left(A_{0}^{*}\right)$ the function $s \rightarrow U^{\odot}(s, t) x^{\odot}$ is the unique weak*-solution to the backward problem (5.8).

Remark 5.10. If $B$ is a Lipschitz continuous mapping from $[0, T]$ to $\mathscr{B}\left(X, X^{\odot *}\right)$, then $B^{*}$ is Lipschitz continuous from $[0, T]$ to $\mathscr{B}\left(X^{\odot}, X^{*}\right)$ and one can prove exactly as in Theorem 4.9 that the assumption $U^{\odot}(s, t) D\left(A_{0}^{*}\right) \subset D\left(A_{0}^{*}\right)$, $(s, t) \in \Delta^{*}$ and $\sup _{0 \leqq s \leqq t}\left\|A_{0}^{*} U^{\odot}(s, t) x^{\odot}\right\|<\infty, x^{\odot} \in D\left(A_{0}^{*}\right), t \in(0, T]$ is automatically satisfied and Theorem 5.9 can be applied. 


\section{Perturbations with fixed finite dimensional range}

In many applications (for instance retarded functional differential equations and age dependent population dynamics) the range of $B(t)$ lies in a fixed finite dimensional subspace of $X^{\odot *}$ for all $t \in[0, T]$. In this section we show in general that in such cases the problem of constructing the evolutionary system $\{U(t, s)\}_{(t, s) \in \Delta}$ reduces to solving a Volterra integral equation on $R^{n}$. Since we do not have an equation for $U^{\odot *}(t, s)$, the only way to obtain $\left\{U^{\odot}(t, s)\right\}_{(t, s) \in \Delta}$ is to take adjoints, restrictions to $X^{\odot}$ and adjoints once more. When the perturbation has finite dimensional range, however, we derive an explicit formula for

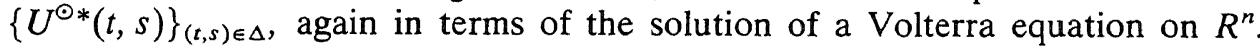
This aspect was not treated in our previous paper [5].

We assume that for all $t \in[0, T]$ the range of $B(t)$ lies in a fixed finite dimensional subspace of $X^{\odot *}$ spanned by $r_{1}^{\odot *}, \ldots, r_{n}^{\odot *}$. Then $B(t)$ has the form

$$
B(t) x=\sum_{i=1}^{n}\left\langle x, r_{i}^{*}(t)\right\rangle r_{i}^{\odot *}
$$

Since we still assume that $B$ is continuous from $[0, T]$ to $\mathscr{B}\left(X, X^{\odot *}\right)$ we require that $r_{i}^{*}$ is continuous from $[0, T]$ to $X^{*}$. We define the entries $q_{i j}(t, u)$ of the matrix-valued function $Q$ by

$$
q_{i j}(t, u)=\left\langle\int_{0}^{u} T_{0}^{\odot *}(\tau) r_{j}^{\odot *} d \tau, r_{i}^{*}(t)\right\rangle, \quad t \in[0, T], \quad u \in[0, T] .
$$

For fixed $t \in[0, T], u \rightarrow q_{i j}(t, u)$ is Lipschitz-continuous on $[0, T]$ and therefore $Q$ has a representation of the form

$$
Q(t, u)=\int_{0}^{u} K(t, \tau) d \tau
$$

where the entries $k_{i j}(t, \tau)$ of $K(t, \tau)$ satisfy $k_{i j}(t, \cdot) \in L^{\infty}[0, T], t \in[0, T]$. Note that

$$
\begin{aligned}
& \left|\frac{q_{i j}\left(t_{1}, u_{2}\right)-q_{i j}\left(t_{1}, u_{1}\right)}{u_{2}-u_{1}}-\frac{q_{i j}\left(t_{2}, u_{2}\right)-q_{i j}\left(t_{2}, u_{1}\right)}{u_{2}-u_{1}}\right| \\
& \quad=\left|\left\langle\frac{1}{u_{2}-u_{1}} \int_{u_{1}}^{u_{2}} T_{0}^{\odot *}(\tau) r_{j}^{\odot *} d \tau, r_{i}^{*}\left(t_{1}\right)-r_{i}^{*}\left(t_{2}\right)\right\rangle\right| \\
& \quad \leqq M \frac{e^{\omega u_{2}}-e^{\omega u_{1}}}{\omega\left(u_{2}-u_{1}\right)}\left\|r_{j}^{\odot *}\right\|\left\|r_{i}^{*}\left(t_{1}\right)-r_{i}^{*}\left(t_{2}\right)\right\| .
\end{aligned}
$$

Hence $t \rightarrow K(t, \cdot)$ is continuous from $[0, T]$ into $L^{\infty}[0, T]$. (As a consequence $t \rightarrow \int_{s}^{t} K(t, t-\tau) h(\tau) d \tau$ is continuous even if $\tau \rightarrow h(\tau)$ is only $L^{x}$.)

It is implied in [5, Lemma 5.1] that if $t \in[0, T]$ and $h$ is a continuous real valued function on $[0, T]$, then

$$
\left\langle\int_{0}^{u} T_{0}^{\odot *}(\tau) r_{j}^{\odot *} h(u-\tau) d \tau, r_{i}^{*}(t)\right\rangle=\int_{0}^{u} k_{i j}(t, u-\tau) h(\tau) d \tau .
$$


Now, as before, let $U(t, s)$ be defined by the variation-of-constants formula

$$
U(t, s) x=T_{0}(t-s) x+\int_{s}^{t} T_{0}^{\odot *}(t-\tau) B(\tau) U(\tau, s) x d \tau, \quad x \in X
$$

and define for every $x \in X$, the $R^{n}$-valued functions $y(\cdot, \cdot ; x)$ and $f(\cdot, \cdot ; x)$ on $\Delta$ by

$$
\begin{aligned}
& y_{i}(t, s ; x)=\left\langle U(t, s) x, r_{i}^{*}(t)\right\rangle, \\
& f_{i}(t, s ; x)=\left\langle T_{0}(t-s) x, r_{i}^{*}(t)\right\rangle .
\end{aligned}
$$

By applying $r_{i}^{*}(t)$ to both sides of (6.5), one obtains

$$
y_{i}(t, s ; x)=f_{i}(t, s ; x)+\sum_{j=1}^{n}\left\langle\int_{s}^{t} T_{0}^{\odot *}(t-\tau) r_{j}^{\odot *} y_{j}(\tau, s ; x) d \tau, r_{i}^{*}(t)\right\rangle .
$$

It follows from (6.4) with $h(\tau)=y_{j}(\tau+s, s ; x), 0 \leqq \tau \leqq t-s$, that

$$
\begin{aligned}
& \left\langle\int_{s}^{t} T_{0}^{\odot *}(t-\tau) r_{j}^{\odot *} y_{j}(\tau, s ; x) d \tau, r_{i}^{*}(t)\right\rangle \\
& \quad=\left\langle\int_{0}^{t-s} T_{0}^{\odot *}(t-s-\tau) r_{j}^{\odot *} y_{j}(\tau+s, s ; x) d \tau, r_{i}^{*}(t)\right\rangle \\
& \quad=\int_{0}^{t-s} k_{i j}(t, t-s-\tau) y_{j}(\tau+s, s ; x) d \tau \\
& \quad=\int_{s}^{t} k_{i j}(t, t-\tau) y_{j}(\tau, s ; x) d \tau .
\end{aligned}
$$

By substituting (6.9) into (6.8), one obtains the Volterra equation

$$
y(t, s ; x)=f(t, s ; x)+\int_{s}^{t} K(t, t-\tau) y(\tau, s ; x) d \tau .
$$

Once $y(t, s ; x)$ is solved from (6.10), the evolutionary system $\{U(t, s)\}_{(t, s) \in \Delta}$ is obtained by substituting $y(t, s ; x)$ into the right-hand side of the variation-ofconstants formula (6.5):

$$
U(t, s) x=T_{0}(t-s) x+\sum_{j=1}^{n} \int_{s}^{t} T_{0}^{\odot *}(t-\tau) r_{j}^{\odot *} y_{j}(\tau, s ; x) d \tau .
$$

Next we derive a Volterra equation and a representation similar to (6.11) for the backward system on $X^{\odot}$. Note that

$$
B^{*}(t) x^{\odot}=\sum_{i=1}^{n}\left\langle r_{i}^{\odot *}, x^{\odot}\right\rangle r_{i}^{*}(t)
$$

and thus the range of $B^{*}(t)$ varies with $t$ and is in general not contained in a fixed finite dimensional subspace of $X^{*}$ for all $t \in[0, T]$. The derivation of the integral equation is therefore slightly more involved in the backward case. 
LemMa 6.1. For any continuous real valued function $h$ on $[0, T]$ the identity

$$
\left\langle r_{j}^{\odot *}, \int_{s}^{t} T_{0}^{*}(\sigma-s) r_{i}^{*}(\sigma) h(\sigma) d \sigma\right\rangle=\int_{s}^{t} k_{i j}(\sigma, \sigma-s) h(\sigma) d \sigma, \quad 0 \leqq s \leqq t \leqq T
$$

holds.

Proof. We first show that

$$
\left\langle\int_{0}^{t} T_{0}^{\odot *}(\tau) x^{\odot *} d \tau, x^{*}\right\rangle=\left\langle x^{\odot *}, \int_{0}^{t} T_{0}^{*}(\tau) x^{*} d \tau\right\rangle
$$

for all $x^{*} \in X^{*}, x^{\odot *} \in X^{\odot *}$. Formula (6.14) obviously holds for $x^{\odot *}=x \in X$. Therefore

$$
\begin{aligned}
& \left\langle x^{\odot *}, \int_{0}^{t} T_{0}^{*}(\tau) x^{*} d \tau\right\rangle \\
& =\lim _{h \downarrow 0} \frac{1}{h}\left\langle\int_{0}^{h} T_{0}^{\odot *}(\sigma) x^{\odot *} d \sigma, \int_{0}^{t} T_{0}^{*}(\tau) x^{*} d \tau\right\rangle \\
& =\lim _{h \downarrow 0} \frac{1}{h}\left\langle\int_{0}^{t} T_{0}^{\odot *}(\tau) \int_{0}^{h} T_{0}^{\odot *}(\sigma) x^{\odot *} d \sigma d \tau, x^{*}\right\rangle \\
& =\lim _{h \downarrow 0} \frac{1}{h}\left\langle\int_{0}^{h} \int_{0}^{t} T_{0}^{\odot *}(\tau+\sigma) x^{\odot *} d \tau d \sigma, x^{*}\right\rangle \\
& =\lim _{h \downarrow 0} \frac{1}{h} \int_{0}^{h}\left\langle\int_{0}^{t} T_{0}^{\odot *}(\tau+\sigma) x^{\odot *} d \tau, x^{*}\right\rangle d \sigma \\
& =\left\langle\int_{0}^{t} T_{0}^{\odot *}(\tau) x^{\odot *} d \tau, x^{*}\right\rangle,
\end{aligned}
$$

since obviously $\sigma \rightarrow\left\langle\int_{0}^{t} T_{0}^{\odot *}(\tau+\sigma) x^{\odot *} d \tau, x^{*}\right\rangle$ is continuous. This proves (6.14).

We now prove (6.13) by noting that the formula holds for $s=t$, and that by integrating both sides with respect to $s$ from $u$ to $t$ one obtains the same result. Indeed, on changing the order of integration and using the definition of $k_{i j}$, one finds

$$
\int_{u}^{t} \int_{s}^{t} k_{i j}(\sigma, \sigma-s) h(\sigma) d \sigma d s=\int_{u}^{t}\left\langle\int_{u}^{\sigma} T_{0}^{\odot *}(s-u) r_{j}^{\odot *} d s, r_{i}^{*}(\sigma)\right\rangle h(\sigma) d \sigma .
$$

On the other hand

$$
\begin{aligned}
\int_{u}^{t}\left\langle r_{j}^{\odot *}, \int_{s}^{t} T_{0}^{*}(\sigma-s) r_{i}^{*}(\sigma) h(\sigma) d \sigma\right\rangle d s & \\
& =\int_{u}^{t}\left\langle r_{j}^{\odot *}, \int_{u}^{\sigma} T_{0}^{*}(\sigma-s) r_{i}^{*}(\sigma) d s\right\rangle h(\sigma) d \sigma .
\end{aligned}
$$

By taking $x^{\odot *}=r_{j}^{\odot *}, x^{*}=r_{i}^{*}(\sigma)$ and $t=\sigma-u$ in (6.14), one finds that the integrals in (6.15) and (6.16) are equal for all $u \in[0, t]$. This proves (6.13). 
Define $z^{T}\left(s, t ; x^{\odot}\right)$ and $g^{T}\left(s, t ; x^{\odot}\right)$ as the row-vectors with components

$$
\begin{aligned}
& z_{i}\left(s, t ; x^{\odot}\right)=\left\langle r_{i}^{\odot *}, U^{\odot}(s, t) x^{\odot}\right\rangle, \\
& g_{i}\left(s, t ; x^{\odot}\right)=\left\langle r_{i}^{\odot *}, T_{0}^{\odot}(t-s) x^{\odot}\right\rangle,
\end{aligned}
$$

where $x^{\odot} \in X^{\odot},(s, t) \in \Delta^{*}, 1 \leqq i \leqq n$. On applying $r_{j}^{\odot *}$ to both sides of the adjoint variation-of-constants formula (5.4), one finds with the aid of Lemma 6.1 that $z^{T}\left(s, t ; x^{\odot}\right)$ satisfies the Volterra equation

$$
z^{T}\left(s, t ; x^{\odot}\right)=g^{T}\left(s, t ; x^{\odot}\right)+\int_{s}^{t} z^{T}\left(\sigma, t ; x^{\odot}\right) K(\sigma, \sigma-s) d \sigma
$$

and that $U^{\odot}(s, t)$ has the representation

$$
U^{\odot}(s, t) x^{\odot}=T_{0}^{\odot}(t-s) x^{\odot}+\sum_{j=1}^{n} \int_{s}^{t} T_{0}^{*}(\sigma-s) r_{j}^{*}(\sigma) z_{j}\left(\sigma, t ; x^{\odot}\right) d \sigma .
$$

Our next objective is to derive a representation of the form (6.11) for the forward system on the big space. Therefore we must give a meaning to $y\left(t, s ; x^{\odot *}\right)$. In order to do so we replace $r_{j}^{\odot *}$ by an arbitrary element $x^{\odot *}$ of $X^{\odot *}$ in the definition (6.2) of $q_{i j}(t, u)$ and call the function so obtained $p_{i}\left(t, u ; x^{\odot *}\right)$. Then $p_{i}\left(t, u ; x^{\odot *}\right)=\int_{0}^{u} m_{i}\left(t, \tau ; x^{\odot *}\right) d \tau$ for some $m_{i}\left(t, \cdot ; x^{\odot *}\right) \in L^{\infty}[0, T]$. Let $f_{i}\left(t, s ; x^{\odot *}\right)=m_{i}\left(t, t-s ; x^{\odot *}\right)$. Then formally $f_{i}\left(t, s ; x^{\odot *}\right)=\left\langle T_{0}^{\odot *}(t-s) x^{\odot *}, r_{i}^{*}(t)\right\rangle$ although this latter expression does not make sense. It is easily seen that $f_{i}\left(\cdot, s ; x^{\odot *}\right)$ is measurable and essentially bounded on $[s, T]$. It follows that the Volterra equation (6.10) with $x$ replaced by $x^{\odot *}$ admits a unique $L^{\infty}$-solution $y\left(t, s ; x^{\odot *}\right)$ on $[s, T]$. In an exactly analogous way we define $g^{T}\left(s, t ; x^{*}\right)$ for all $x^{*} \in X^{*}$ and conclude that equation (6.19) with $x^{\odot}$ replaced by $x^{*}$ has a unique $L^{\infty}$-solution $z^{T}\left(s, t ; x^{*}\right)$ on $[0, T]$.

It remains to show that we can represent $U^{\odot *}(t, s) x^{\odot *}$ in terms of $y\left(t, s ; x^{\odot *}\right)$ and $U^{*}(s, t)$ in terms of $z^{T}\left(s, t ; x^{*}\right)$. It turns out to be convenient to express $y\left(t ; s ; x^{\odot *}\right)$ and $z^{T}\left(s, t ; x^{*}\right)$ with the aid of the resolvent. Let $R(t, s)$ be the resolvent kernel corresponding to the kernel $K(t, t-s)$. By definition, $R(t, s)$ is the solution of the matrix equation

$$
R(t, s)=K(t, t-s)+\int_{s}^{t} R(t, \tau) K(\tau, \tau-s) d \tau
$$

or, equivalently,

$$
R(t, s)=K(t, t-s)+\int_{s}^{t} K(t, t-\tau) R(\tau, s) d \tau
$$

Note that the $(i, j)$ element of $R(t, s)$ is given by $y_{i}\left(t, s ; r_{j}^{\odot *}\right)$. One has the explicit representations

$$
y\left(t, s ; x^{\odot *}\right)=f\left(t, s ; x^{\odot *}\right)+\int_{s}^{t} R(t, \tau) f\left(\tau, s ; x^{\odot *}\right) d \tau
$$

and

$$
z^{T}\left(s, t ; x^{*}\right)=g^{T}\left(s, t ; x^{*}\right)+\int_{s}^{t} g^{T}\left(\sigma, t ; x^{*}\right) R(\sigma, s) d \sigma,
$$

see [18, Chap. 4] or [13]. 
We are now ready to state the main result of this section.

THEOREM 6.2. Let $y\left(t, s ; x^{\odot *}\right)$ and $z^{T}\left(s, t ; x^{*}\right)$ be the unique $L^{\infty}$-solutions of the Volterra equations

$$
\begin{aligned}
y\left(t, s ; x^{\odot *}\right)= & f\left(t, s ; x^{\odot *}\right) \\
& +\int_{s}^{t} K(t, t-\tau) y\left(\tau, s ; x^{\odot *}\right) d \tau, \quad t \in[s, T],
\end{aligned}
$$

and

$$
\begin{aligned}
z^{T}\left(s, t ; x^{*}\right)= & g^{T}\left(s, t ; x^{*}\right) \\
& +\int_{s}^{t} z^{T}\left(\sigma, t ; x^{*}\right) K(\sigma, \sigma-s) d \sigma, \quad s \in[0, T],
\end{aligned}
$$

respectively. Then

$$
\begin{aligned}
U^{\odot *}(t, s) x^{\odot *} & =T_{0}^{\odot *}(t-s) x^{\odot *}+\sum_{j=1}^{n} \int_{s}^{t} T_{0}^{\odot *}(t-\tau) r_{j}^{\odot *} y_{j}\left(\tau, s ; x^{\odot *}\right) d \tau, \\
U^{*}(s, t) x^{*} & =T_{0}^{*}(t-s) x^{*}+\sum_{j=1}^{n} \int_{s}^{t} T_{0}^{*}(\sigma-s) r_{j}^{*}(\sigma) z_{j}\left(\sigma, t ; x^{*}\right) d \sigma,
\end{aligned}
$$

for all $x^{\odot *} \in X^{\odot *}, x^{*} \in X^{*}, 0 \leqq s \leqq t \leqq T$.

Proof. For $x^{\odot} \in X^{\odot}$ one has

$$
\begin{aligned}
& \left\langle\sum_{j=1}^{n} \int_{s}^{t} T_{0}^{\odot *}(t-\tau) r_{j}^{\odot *} y_{j}\left(\tau, s ; x^{\odot *}\right) d \tau, x^{\odot}\right\rangle \\
& \quad=\int_{s}^{t} g^{T}\left(\tau, t ; x^{\odot}\right) y\left(\tau, s ; x^{\odot *}\right) d \tau \\
& \quad=\int_{s}^{t} g^{T}\left(\tau, t ; x^{\odot}\right)\left[f\left(\tau, s ; x^{\odot *}\right)+\int_{s}^{\tau} R(\tau, \sigma) f\left(\sigma, s ; x^{\odot *}\right) d \sigma\right] d \tau \\
& \quad=\int_{s}^{t}\left[g^{T}\left(\sigma, t ; x^{\odot}\right)+\int_{\sigma}^{t} g^{T}\left(\tau, t ; x^{\odot}\right) R(\tau, \sigma) d \tau\right] f\left(\sigma, s ; x^{\odot *}\right) d \sigma \\
& \quad=\int_{s}^{t} z^{T}\left(\sigma, t ; x^{\odot}\right) f\left(\sigma, s ; x^{\odot *}\right) d \sigma .
\end{aligned}
$$

On the other hand,

$$
\begin{aligned}
& \left\langle U^{\odot *}(t, s) x^{\odot *}-T_{0}^{\odot *}(t-s) x^{\odot *}, x^{\odot}\right\rangle \\
& \quad=\left\langle x^{\odot *}, \sum_{j=1}^{n} \int_{s}^{t} T_{0}^{*}(\sigma-s) r_{j}^{*}(\sigma) z_{j}\left(\sigma, t ; x^{\odot}\right) d \sigma\right\rangle \\
& =\lim _{h \downarrow 0}\left\langle\frac{1}{h} \int_{0}^{h} T_{0}^{\odot *}(\tau) x^{\odot *} d \tau, \sum_{j=1}^{n} \int_{s}^{t} T_{0}^{*}(\sigma-s) r_{j}^{*}(\sigma) z_{j}\left(\sigma, t ; x^{\odot}\right) d \sigma\right\rangle \\
& =\lim _{h \downarrow 0} \int_{s}^{t} z^{T}\left(\sigma, t ; x^{\odot}\right) f\left(\sigma, s ; \frac{1}{h} \int_{0}^{h} T_{0}^{\odot *}(\tau) x^{\odot *} d \tau\right) d \sigma \\
& =\int_{s}^{t} z^{T}\left(\sigma, t ; x^{\odot}\right) f\left(\sigma, s ; x^{\odot *}\right) d \sigma,
\end{aligned}
$$


where the last equality follows from the dominated convergence theorem and the fact that

$$
\begin{aligned}
& f_{i}\left(\sigma, s ; \frac{1}{h} \int_{0}^{h} T_{0}^{\odot *}(\tau) x^{\odot *} d \tau\right) \\
& \quad=\left\langle T_{0}(t-s) \frac{1}{h} \int_{0}^{h} T_{0}^{\odot *}(\tau) x^{\odot *} d \tau, r_{i}^{*}(t)\right\rangle=\frac{1}{h}\left\langle\int_{0}^{h} T_{0}^{\odot *}(\tau+t-s) x^{\odot *} d \tau, r_{i}^{*}(t)\right\rangle \\
& \quad=\frac{1}{h}\left[p_{i}\left(t, t-s+h ; x^{\odot *}\right)-p_{i}\left(t, t-s ; x^{\odot *}\right) \rightarrow f_{i}\left(t, s ; x^{\odot *}\right)\right. \text { almost everywhere. }
\end{aligned}
$$

as $h \downarrow 0$ by the definition of $f\left(t, s ; x^{\odot *}\right)$. The formulae (6.29) and (6.30) together imply (6.27). Formula (6.28) is proved in exactly the same way.

To conclude this section we identify the kernel, forcing function and solution of the Volterra equation (6.10) in the application to the retarded functional differential equation (1.6) and show that the forward evolutionary system corresponding to the abstract problem is the evolutionary system corresponding to equation (1.6).

The element $r_{i}^{*}(t)$ is the $i$ th row of $\zeta(t, \cdot)$ and $r_{i}^{\odot *}=\left(e_{i}, 0\right)$, where $e_{i}$ denotes the $i$ th unit column vector in $R^{n}$. It is convenient to combine these into matrices $r^{*}(t)=\zeta(t, \cdot)$ and $r^{\odot *}=(I, 0)$, where $I$ is the identity matrix. Then

$$
T_{0}^{\odot *}(t) r^{\odot *}=(I, H(t+\cdot) I)
$$

Substituting (6.31) into (6.11) one finds

$$
\begin{aligned}
{[U(t, s) \phi](\theta)=} & {\left[T_{0}(t-s) \phi\right](\theta) } \\
& +\int_{s}^{\max \{s, t+\theta\}} y(\tau, s ; \phi) d \tau, \quad \theta \in[-1,0] .
\end{aligned}
$$

Define $x(t ; \phi)=[U(t, s) \phi](0)$. Then (6.32) implies that $x(t ; \phi)=\phi(0)+$ $\int_{s}^{t} y(\tau, s ; \phi) d \tau$ and hence

$$
\dot{x}(t ; \phi)=y(t, s ; \phi) .
$$

It follows from (6.32) and (6.33) that

$$
\begin{aligned}
{[U(t, s) \phi](\theta) } & =\phi(0)+\int_{s}^{t+\theta} y(\tau, s ; \phi) d \tau \\
& =x(t+\theta, \phi), \quad t+\theta \geqq s .
\end{aligned}
$$

On using (6.6) and (6.34) one finds that

$$
y(t, s ; \phi)=\left\langle U(t, s) \phi, r^{*}(t)\right\rangle=\int_{0}^{1} x(t-\theta ; \phi) d_{\theta} \zeta(t, \theta) .
$$

Formulae (6.33)-(6.35) show that $\{U(t, s)\}_{(t, s) \in \Delta}$ is indeed the evolutionary system corresponding to the solution to (1.6). We remark that we have only considered the forward problem and therefore we have not made use of the norm continuity of $r^{*}(t)$. Thus everything applies to the problem considered in Example 3.3. 
It follows from (6.31) that

$$
\begin{aligned}
q_{i j}(t, u) & =\left\langle\int_{0}^{u} T_{0}^{\odot *}(\tau) r_{j}^{\odot *} d \tau, r_{i}^{*}(t)\right\rangle \\
& =\int_{0}^{u}(u-\theta) d_{\theta} \zeta_{i j}(t, \theta) \\
& =\int_{0}^{u} \zeta_{i j}(t, \theta) d \theta,
\end{aligned}
$$

and hence $K=\zeta$. The forcing function is obtained as follows:

$$
\begin{aligned}
f(t, s ; \phi) & =\left\langle T_{0}(t-s) \phi, r^{*}(t)\right\rangle \\
& =\int_{0}^{1}\left[T_{0}(t-s) \phi\right](-\theta) d_{\theta} \zeta(t, \theta) \\
& =\int_{t-s}^{1} \phi(t-s-\theta) d_{\theta} \zeta(t, \theta)+\zeta(t, t-s) \phi(0) .
\end{aligned}
$$

Note that the fundamental matrix solution of [14, Chap. 6] is, in our setting, equal to $U^{\odot *}(t, s) r^{\odot *}$ and therefore corresponds to the resolvent of the Volterra equation.

Finally, we point out that the action of $r^{\odot *}$ corresponds to taking the limit from above in zero. Therefore for $s<t$ we have $g^{T}\left(s, t ; x^{*}\right)=x^{*}((t-s)+)=x^{*}(t-s)$. In other words, the forcing function in the Volterra equation and the dual state $x^{*}$ are one and the same thing! One can consequently interpret the action of $U^{*}(s, t)$ in terms of transformations of the forcing function in the Volterra equation (see [10] and the references given there for a more detailed elaboration in the autonomous case).

\section{Acknowledgment}

Four of the five authors took part in the 1986 Texel Workshop on Models for Physiologically Structured Populations. The discussions during the workshop have strongly stimulated cooperation.

\section{References}

1 H. Amann. Dual semigroups and second order linear elliptic boundary value problems. Israel J. Math. 45 (1983), 225-254.

2 J. M. Ball. Strongly continuous semigroups, weak solutions, and the variation of constants formula. Proc. Amer. Math. Soc. 63 (1977), 370-373.

3 A. Bellini-Morante. Applied Semigroups and Evolution Equations (Oxford: Clarendon Press, 1979).

4 P. L. Butzer and H. Berens. Semi-groups of operators and approximation (Berlin: Springer, 1967).

5 Ph. Clément, O. Diekmann, M. Gyllenberg, H. J. A. M. Heijmans and H. R. Thieme Perturbation theory for dual semigroups I. The sun-reflexive case. Math. Ann. 277 (1987), $709-725$.

6 Ph. Clément, H. J. A. M. Heijmans et al. One-parameter semigroups. CWI Monographs (Amsterdam: North-Holland, 1987). 
7 W. Desch and W. Schappacher. On relatively bounded perturbations of linear $C_{0}$-semigroups. Ann. Scuola Norm. Sup. Pisa Cl. Sci. (4) 11 (1984), 327-341.

8 W. Desch, W. Schappacher and Kang Pei Zhang. Semilinear evolution equations (preprint).

9 W. Desch, I. Lasiecha and W. Schappacher. Feedback boundary control problems for linear semigroups. Israel J. Math. 51 (1985), 177-207.

10 O. Diekmann. Perturbed dual semigroups and delay equations. In Dynamics of infinite dimensional systems (S-N. Chow and J. K. Hale, eds.) NATO ASI Series, Vol. F37, 67-73 (Berlin: Springer, 1987).

11 J. Dieudonné. Foundations of modern analysis (New York: Academic Press, 1969).

12 J. R. Dorroh and R. A. Graff. Integral equations in Banach spaces, a general approach to the linear Cauchy problem, and applications to the nonlinear problem. J. Integral Equations 1 (1979), 309-359.

13 G. Gripenperg, S-O. Londen and O. Staffans. Volterra equations (in preparation).

14 J. K. Hale. Theory of functional differential equations (New York: Springer, 1977).

15 E. Hille and R. S. Phillips. Functional analysis and semi-groups (Providence: Amer. Math. Soc., 1957).

16 T. Kato. Integration of the equation of evolution in a Banach space. J. Math. Soc. Japan 5 (1953), 208-234.

17 H. Kellermann. Linear evolution equations with time-dependent domain. Semesterbericht Funktionalanalysis, Tübingen, Wintersemester 1985/86.

18 R. K. Miller. Nonlinear Volterra integral equations (Menlo Park: Benjamin, 1971).

19 A. Pazy. Semigroups of linear operators and applications to partial differential equations (New York: Springer, 1983).

20 W. Rudin. Principles of mathematical analysis, Third Edition (New York: McGraw-Hill, 1976).

21 H. Tanabe. Equations of Evolution (London: Pitman, 1979).

(Issued 21 June 1988) 